\title{
C-3ß-Tethered Functional Cholesterol Conjugates by Nitrile Oxide Alkyne Cycloaddition (NOAC)
}

\author{
Virginie Algay, ${ }^{[a]}$ Justine O'Sullivan, ${ }^{[a]}$ and Frances Heaney*[a]
}

Keywords: Steroids / Lipids / Cycloaddition / Alkynes / Heterocycles / Conjugation

Covalent cholesterol conjugates have been prepared by isoxazole-generating [3+2] nitrile oxide alkyne cycloaddition (NOAC) chemistry. Steroidal building blocks functionalised with either an alkyne or a nitrile oxide precursor were evalu- ated. The reaction has been demonstrated for the tethering to cholesterol of groups capable of bioreporting, and for the formation of cholesterol-biomolecule conjugates.

\section{Introduction}

Cholesterol is one of the most common natural lipids, and due to its bioavailability and structural rigidity, functionalised cholesterol building blocks have attracted interest in areas ranging from materials to medicinal chemistry. There have been a number of reports on triazole-mediated conjugation by copper(I)-promoted dipolar cycloaddition using both azido lipids and cholesterol-alkynes. This powerful click chemistry approach ${ }^{[1]}$ has opened access to new cholesterol pharmacophores, ${ }^{[2 \mathrm{a}, 2 \mathrm{~b}]}$ and has allowed the creation of specialist materials, including cholesterol-incorporating organogels, ${ }^{[2 \mathrm{c}, 2 \mathrm{~d}]}$ nanoparticles, ${ }^{[2 \mathrm{e}]}$ liquid crystals, ${ }^{[2]}$ and orthopaedic medical devices. ${ }^{[2 \mathrm{~g}]}$ It has also facilitated access to cholesterol-dye conjugates for biomembrane ${ }^{[2 \mathrm{~h}]}$ or metabolic studies, ${ }^{[2 \mathrm{i}]}$ to steroidal glycosides, ${ }^{[2 \mathrm{j}]}$ and to lipid-oligonucleotide conjugates for drug delivery applications. ${ }^{[2 \mathrm{k}-2 \mathrm{~m}]}$ In addition, catalyst-free routes to triazole-linked cholesterol-oligonucleotide conjugates have been reported. ${ }^{[3]}$ However, aside from exploitation of the masked functionality of the isoxazole nucleus to access polyhydroxylated steroids, cholesterol conjugates with this heterocyclic linker are relatively unknown. Thermally promoted cycloadditions to $\mathrm{C}-17$-anchored steroidal alkynes have yielded important reaction intermediates. ${ }^{[4]}$ In recent years, the regioselectivity of such cycloadditions has been enhanced through the use of copper ${ }^{[5 \mathrm{a}-5 \mathrm{c}]}$ or ruthenium catalysis. ${ }^{[5 \mathrm{~d}]}$ Transient nitrile oxide dipoles have been generated by the action of $\mathrm{PhNCO}$ on steroidal nitroalkane precursors, ${ }^{[6]}$ or from C-22 aldoximes by the NCS/ $/ \mathrm{NEt}_{3}$ protocol. ${ }^{[7]}$ The work described in this paper demonstrates the versatility of nitrile oxide alkyne cycloaddition (NOAC)

[a] Department of Chemistry, National University of Ireland, Maynooth, Co. Kildare, Republic of Ireland

E-mail: mary.f.heaney@nuim.ie http://www.nuim.ie/chemistry

Supporting information for this article is available on the WWW under http://dx.doi.org/10.1002/ejoc.201301822. chemistry ${ }^{[8]}$ for the formation of cholesterol conjugates anchored by way of a polar, aromatic, metabolically stable isoxazole nucleus.

\section{Results and Discussion}

To maximize structural diversity, and to avoid potential stereochemical complications, we required the alkyne or nitrile oxide precursors to be anchored to the $\mathrm{C}-3 \beta$ oxygen atom rather than directly to the tetracyclic core. ${ }^{[6 b]}$ The Montmorillonite K-10 (MK-10) ${ }^{[9]}$ promoted route to steroidal ethers 1-3 is shown in Scheme 1. The reported procedure for the formation of propargyl ether $\mathbf{1}$, which requires a solution of cholesterol and the corresponding alcohol in $\mathrm{CHCl}_{3}$ to be stirred for $7 \mathrm{~d}$ at $55^{\circ} \mathrm{C}^{[10]}$ was modified so that the product could be obtained with minimal compromise in yield after just $17 \mathrm{~h}$ at $90{ }^{\circ} \mathrm{C}$ in a microwave (MW) reactor. Following the modified procedure, homologous alkynyl ethers $\mathbf{2}$ and $\mathbf{3}$ were obtained in 62 and $70 \%$ yields, respectively.

Model NOAC reactions were explored between 1-3 and phenyl nitrile oxide, which was generated in situ from benzaldehyde oxime on exposure to an ethanolic solution of chloramine- $\mathrm{T}$ (Ch-T). While there was no evidence for a compromise in regiochemical integrity, the yields of 3,5-disubstituted isoxazoles $\mathbf{4 a - 6 a}$ were greatly influenced by the spacing between the bulky lipid and the reacting alkyne. Thus, compound 6a, with a 4-carbon linker, was obtained in $90 \%$ yield after $1 \mathrm{~h}$ at room temperature. Compound $\mathbf{5 a}$, with a shorter ethylene spacer, was obtained in only $44 \%$ after the same reaction time. Significantly, compound $\mathbf{4 a}$, with a methylene spacer, was formed in a mere $19 \%$ yield, even after $17 \mathrm{~h}$. The same reactivity trend was seen with the putative dipoles generated from 1-naphthyl and 1-pyrenyl oximes. Gratifingly, we found that the sluggish reactivity of propargylated steroid 1a could be circumvented by conducting the reaction in a microwave reactor. In this way, 


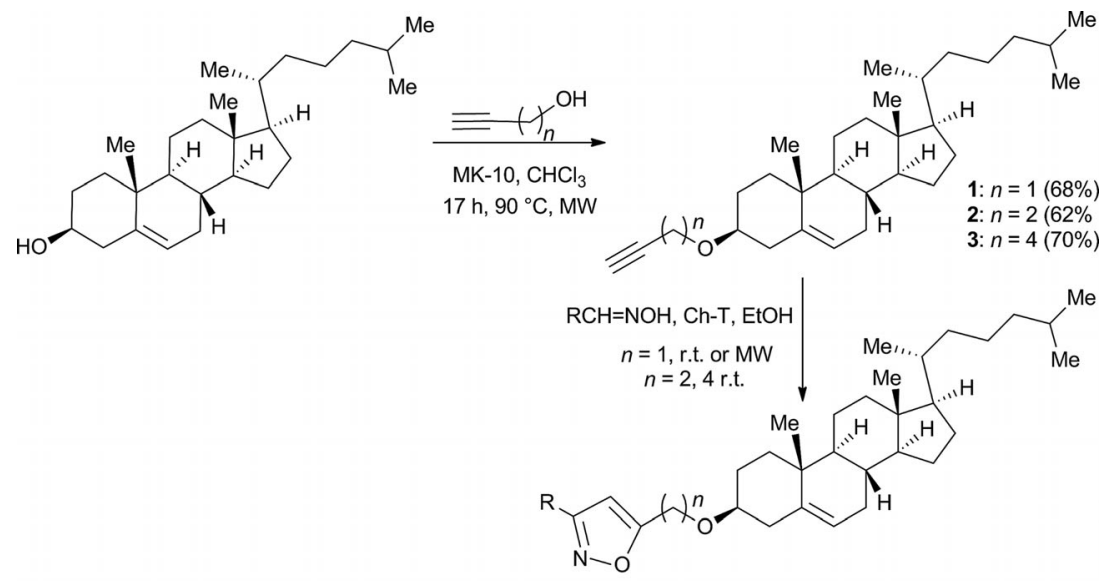

$\begin{array}{lll}\text { a: } \mathrm{R}=\mathrm{Ph} & \mathbf{4 a}: n=1\left(19 \%, \mathrm{MW}, 100^{\circ} \mathrm{C} 1 \mathrm{~h}, 78 \%\right) ; \mathbf{5 a}: n=2(44 \%) ; \mathbf{6 a}: n=4(90 \%) \\ \text { b: } \mathrm{R}=1 \text {-naphthyl } & \text { 4b: } n=1\left(43 \%, \mathrm{MW}, 60^{\circ} \mathrm{C}, 0.5 \mathrm{~h}, 91 \%\right) ; \mathbf{5 b}: n=2(54 \%) ; \mathbf{6 b}: n=4(90 \%) \\ \text { c: } \mathrm{R}=1 \text {-pyrenyl } & \text { 4c: } n=1\left(17 \%, \mathrm{MW}, 60^{\circ} \mathrm{C}, 0.5 \mathrm{~h}, 71 \%\right) ; \mathbf{5 c}: n=2(35 \%) ; 6 \mathbf{c}: n=4(80 \%)\end{array}$

Scheme 1. Steroidal alkynes and conjugates formed by NOAC chemistry.

the product yields for $\mathbf{4 a}-\mathbf{4 c}$ increased to 78,96 , and $71 \%$, respectively.

The potential of the NOAC reaction to access biologically relevant cholesterol probes is illustrated by the preparation of steroid-coumarin $\mathbf{7}$ and steroid-azobenzene $\mathbf{8}$ conjugates. Cholesterol is implicated in a vast array of biological processes, and fluorescent derivatives are useful tools that can be used for imaging in cells and tissues; ${ }^{[11]}$ azoderivatised molecules are attractive for the construction of thermal and photoresponsive materials, ${ }^{[12 a, 12 b]}$ including phototriggered drug-delivery vehicles. ${ }^{[12 c]}$ Fluorescent coumarin conjugate 7 was formed in $75 \%$ yield by the cycloaddition reaction between 3 and the nitrile oxide generated from coumarin 6-aldoxime. ${ }^{[13 a]}$ Photoresponsive azo-conjugated steroid $\mathbf{8}$ was obtained by a parallel reaction sequence involving 4-(phenylazo)benzaldehyde oxime ${ }^{[13 \mathrm{~b}]}$ as the dipole precursor (Figure 1). Initially obtained almost exclusively as the trans-isomer, 8 was transformed into an 84:16 mixture of trans and cis isomers after exposure to sunlight for $2 \mathrm{~h}$. When the sample was returned to darkness, the original exclusively trans geometry was restored.

To increase the flexibility of the NOAC approach to cholesterol conjugation, steroidal building blocks bearing nitrile oxide precursors were pursued. The reported successes of cholesterol chloroformate as a handle for the introduction of alkyne ${ }^{[14 a]}$ or azido ${ }^{[14 b]}$ functionalities suggested that it would be an appropriate building block for oxime introduction by way of an amidocarbamate linker. However, after sequential acetal deprotection and oxime formation, as outlined in Scheme 2, the target oxime (i.e., 12) was obtained in just $2 \%$ overall yield, primarily as a result of a low-yielding deprotection step. Fortunately, we discovered that aldehyde regeneration and oximation could be achieved in a one-pot procedure. Thus, direct exposure of acetal 10 to $\mathrm{NH}_{2} \mathrm{OH} \cdot \mathrm{HCl}$ in ethanolic pyridine in a microwave reactor $\left(125^{\circ} \mathrm{C}, 1 \mathrm{~h}, P_{\max } 300 \mathrm{~W}\right)$ yielded steroidal oxime $\mathbf{1 2}$ in $91 \%$ yield. Putative steroidal nitrile oxide 13, derived from 12 upon treatment with chloramine-T, was

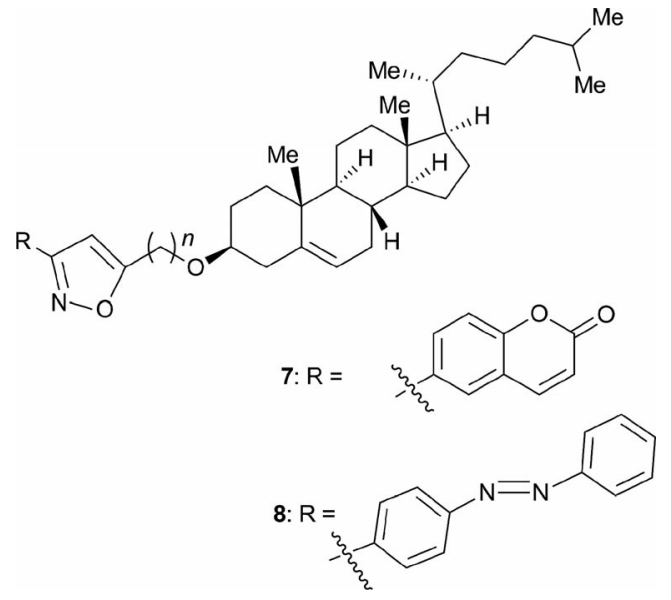

Figure 1. Structures of fluorescent and photoresponsive cholesterol conjugates 7 and $\mathbf{8}(n=4)$.

trapped with both propargyl alcohol and propargyl phenyl ether, however, the yields of the cholesterol conjugates (i.e., 14a and 14b) were modest ( 21 and $37 \%$ ). The low yields of the cycloadducts may be a consequence of solubility issues arising from a suboptimal balance of hydrophilic and hydrophobic character of the microenvironments of the lipid-amidocarbamate-isoxazole conjugate. Stable longchain hydrophilic linkers are attractive for bioconjugation, and for this reason, amidocarbamate substrates were abandoned in favour of ether-linked analogues $\mathbf{1 6}$ (Scheme 3). Regioisomeric steroidal oxime ethers $\mathbf{1 6}$ were prepared in two steps, as shown in Scheme 3. MK-10-mediated condensation $^{[9]}$ between cholesterol and the relevant hydroxyethoxybenzaldehyde gave aldehydes $\mathbf{1 5}$ in 35-58\% yield after $8 \mathrm{~h}$ in a microwave reactor $\left(P_{\max } 300 \mathrm{~W}\right)$. As noted for $\boldsymbol{p} \mathbf{- 1 5}$, no significant increase in yield was observed when the reaction time was doubled to $17 \mathrm{~h}$. Oximation proceeded quantitatively, and the latent reactivity of $\mathbf{1 6}$ as a nitrile oxide precursor was demonstrated by the formation of cholesterol conjugates 18. Thus, following overnight stirring of an eth- 


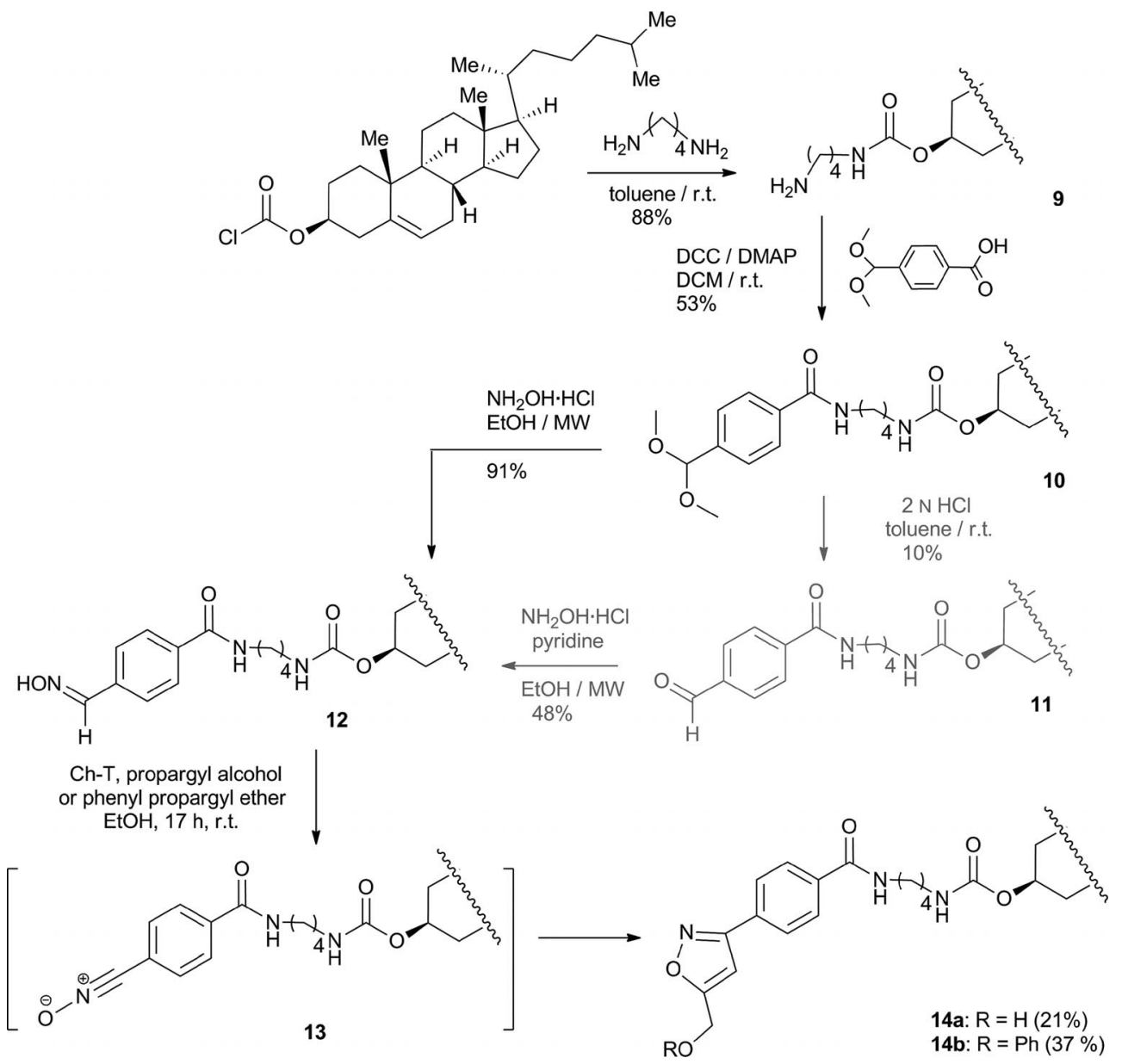

Scheme 2. Amidocarbamate steroidal oximes and conjugates formed by NOAC chemistry. DCC $=N, N^{\prime}$-dicyclohexylcarbodiimide; DMAP = 4-(dimethylamino)pyridine.

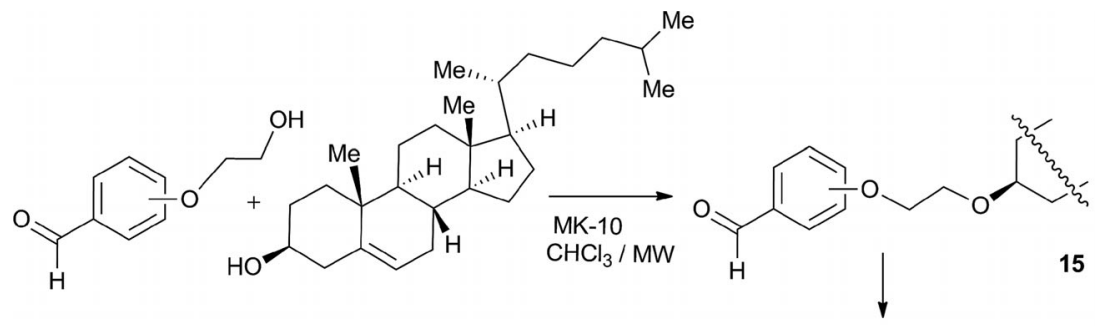

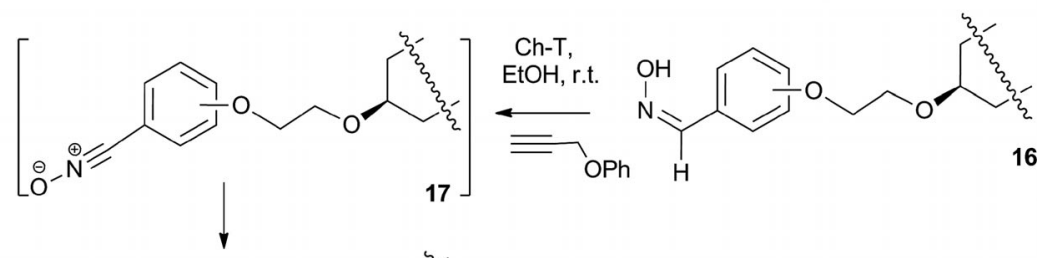<smiles>[Y]CC(COCCOc1ccccc1)OCCOc1cc(-c2ccccc2)on1</smiles>

Scheme 3. Steroidal oximes and conjugates formed by NOAC chemistry. 


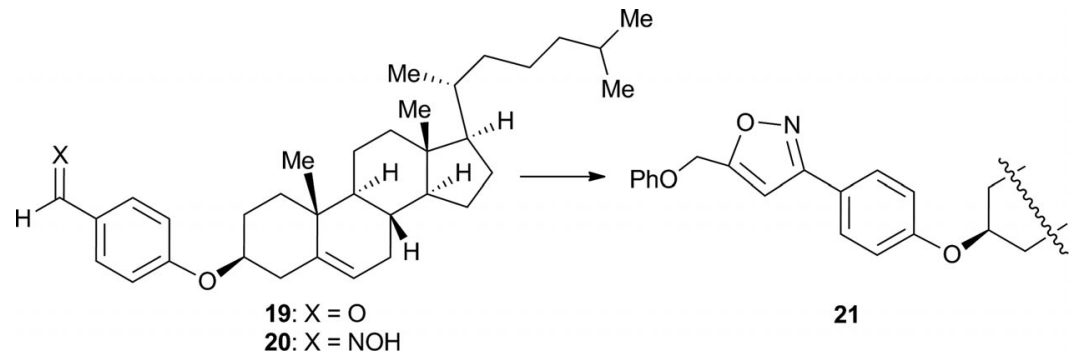

Scheme 4. Aryl steroidal ethers and conjugate formation by NOAC chemistry.

anolic solution of oxime $\mathbf{1 6}$ and chloramine- $\mathrm{T}$ in the presence of phenyl propargyl ether, 18a-18c were isolated in 29$58 \%$ yield. It is likely that the variation in yield of the $o-$, $m$-, and $p$-substituted isomeric cycloadducts reflects the effect of the bulky steroidal core on the transition state leading to isoxazole formation. Thus, the steric demand is greatest for the alignment leading to $\boldsymbol{o - 1 8}$, and the yield of the cycloadduct is lowest (29\%); the bulk of the steroidal core has a minimal impact on the transition state leading to $\boldsymbol{p}$ 18, which results in a significantly improved yield of the cycloadduct (58\%). A further improvement in the yield of $\boldsymbol{p}$-18 to an attractive $80 \%$ was achieved by adding the steroidal oxime and chloramine-T portionwise to a solution of the dipolarophile in ethanol.

Aryl ethers of natural alcohols are inherently interesting both as end products and as intermediates en route to functional materials. Their synthesis is non-trivial and has attracted significant interest. ${ }^{[15]}$ To validate NOAC chemistry as a vehicle for the assembly of cholesterol conjugates like 21, aldehyde-functionalised aryl ether $\mathbf{1 9}$ was required. It is known that MK-10-promoted aryl etherification of cholesterol in $\mathrm{CHCl}_{3}$ at $50-70{ }^{\circ} \mathrm{C}$ is suited to electron-rich, but not to electron-deficient phenols. ${ }^{[9]}$ However, we are pleased to report that the use of microwave irradiation in the reaction between cholesterol and $p$-hydroxybenzaldehyde $\left(90^{\circ} \mathrm{C}, 8 \mathrm{~h}, P_{\max } 300 \mathrm{~W}\right)$ resulted in the formation of 19 $(62 \%)$. With the key intermediate to hand, subsequent oximation and cycloaddition resulted in the formation of isoxazole-linked aryl cholesterol ether 21 (Scheme 4).

Covalently functionalised cholesterol bioconjugates including sugar-cholesterol, ${ }^{[16 a]}$ amphiphilic DNA-cholester$\mathrm{ol},{ }^{[16 b]}$ and nucleoside-cholesterol ${ }^{[16 c]}$ conjugates have valuable roles as components of model biological membranes, ${ }^{[16 \mathrm{~d}]}$ and as specialist materials for drug-delivery applications. Thus, the potential of NOAC chemistry to deliver cholesterol bioconjugates is an effective marker of its utility. Steroidal glycoconjugate formation is demonstrated by the regioselective formation of $\mathbf{2 3}(85 \%)$ by the reaction of putative nitrile oxide $\boldsymbol{p}$-17 with protected D-galactose $\mathbf{2 2}$ as a model sugar dipolarophile. ${ }^{[17]}$ The selective tethering of one or two cholesterol units to a thymidine skeleton was demonstrated by trapping of the same dipole by $5^{\prime}$-protected mono- or bis-propargylated thymidines $\mathbf{2 4}$ or $\mathbf{2 6}^{[18]}$ to give conjugates $\mathbf{2 5}$ and $\mathbf{2 7}$ in 81 and $68 \%$ yields, respectively (Scheme 5). The mono- and bis-steroidal conjugates are potentially useful building blocks for the formation of syn- thetic oligonucleotides with tuneable amphiphilic properties.
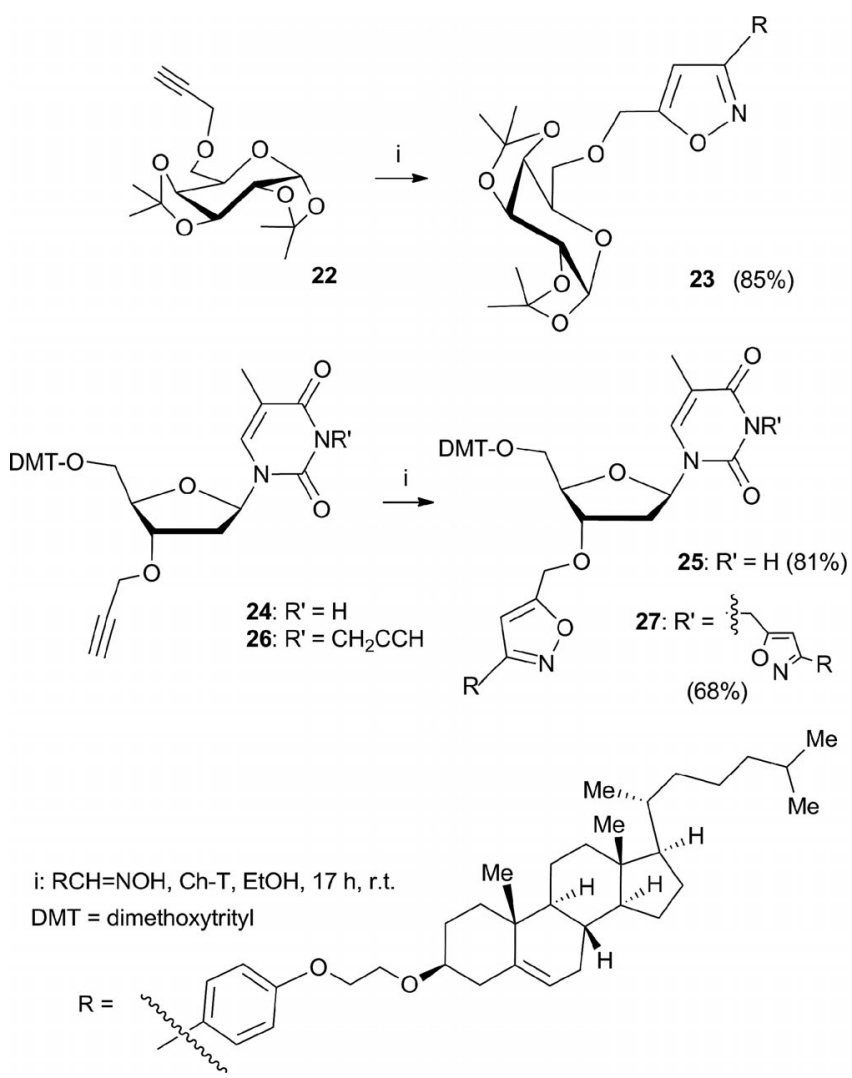

Scheme 5. Cholesterol-biomolecule conjugates formed by NOAC chemistry.

The structures of all the isoxazole-linked cholesterol conjugates are supported by spectroscopic data. In particular, diagnostic NMR spectral signals consistent with the regiospecific formation of 3,5-disubstituted isoxazoles include a singlet resonance in the region $6.30-6.63 \mathrm{ppm}$ of the ${ }^{1} \mathrm{H}$ spectrum, characteristic of the $4-\mathrm{H}$ ring proton, ${ }^{[5 \mathrm{~d}]}$ and a resonance in the of the ${ }^{13} \mathrm{C}$ spectrum in the range 99 $104 \mathrm{ppm}$ due to the corresponding carbon atom.

\section{Conclusions}

In conclusion, NOAC chemistry has been verified as a straightforward and convenient method for the formation of diverse cholesterol conjugates including those of poten- 
tial value as biochemical probes. The NOAC tethering method avoids catalysts and optimisation of numerous reagents as well as the potentially problematic task of separating such reagents from the crude reaction products. The reaction is flexible in that either of the two functionalities needed for the [3+2] cycloaddition can be introduced at the $3 \beta$ position of the steroidal core. This facilitates the introduction of the conjugating partner as either the dipole or the dipolarophile, without incurring any stereochemical penalties. The hydrophobic/hydrophilic balance of the steroidal reactants and products is important for the success of the reaction, as is the length of the linker that separates the rigid cholesterol core from the cycloaddition partner. In a future communication, we will describe the use of NOAC on the solid phase for the synthesis of oligonucleotidecholesterol conjugates.

\section{Experimental Section}

General Remarks: Analytical TLC was carried out on precoated $(250 \mathrm{~mm})$ silica gel $60 \mathrm{~F}-254$ plates from Merck. Plates were visualized by UV irradiation, and/or staining with $\mathrm{H}_{2} \mathrm{SO}_{4}(5 \%$ in ethanol) followed by heating. Flash-chromatography-grade silica gel 60 (230-400 mesh) was obtained from Merck. High-resolution mass spectra were recorded with a Bruker BioApex $70 \mathrm{eV}$ spectrometer. IR spectra were recorded on disposable polyethylene cards $(19 \mathrm{~mm}$, type 61,3M) with a Perkin-Elmer Spectrum 100 RX-I series FTIR spectrophotometer. Reactions using microwave (MW) irradiation were carried out with a Discover SP (CEM, Matthews North Carolina, USA) apparatus, with an Explorer, controlled by the Synergy ${ }^{\mathrm{TM}}$ software. Melting points were determined with a Stuart Equipment SMP 11 apparatus. NMR spectra were obtained for ${ }^{1} \mathrm{H}$ at $300 \mathrm{MHz}$ and for ${ }^{13} \mathrm{C}$ at $75 \mathrm{MHz}$ with a Bruker instrument at $25^{\circ} \mathrm{C}$. All samples for NMR spectroscopy were prepared in $\mathrm{CDCl}_{3}$. Chemical shifts are reported in ppm downfield from tetramethylsilane, and coupling constants are reported in Hertz. Anhydrous solvents were distilled from calcium chloride, calcium hydride, potassium hydroxide, or sodium as appropriate. Chemical reagents were purchased from Aldrich Chemical Company unless otherwise noted, and were used without further purification.

General Etherification Procedure: A mixture of cholesterol (500 mg, $1.29 \mathrm{mmol})$, alkynyl alcohol $(6.47 \mathrm{mmol}, 5$ equiv.), and MK-10 ( $1.00 \mathrm{~g}$, activated at $120^{\circ} \mathrm{C}$ overnight before use) in chloroform $(5 \mathrm{~mL})$ was heated to $90^{\circ} \mathrm{C}$ for $17 \mathrm{~h}$ in a microwave reactor $(10 \mathrm{~mL}$ vessel, $\left.P_{\max }=300 \mathrm{~W}\right)$. The solvent was removed under reduced pressure, hexane $(20 \mathrm{~mL})$ was added, and the catalyst was removed by filtration and then washed with hexane. The filtrate and washings were evaporated, and the residue was purified by flash column chromatography (hexane/EtOAc; $0-5 \%$ ) to give the title compounds as white solids.

(3ß)-3-(2-Propynyloxy)cholest-5-ene (1): ${ }^{[10]}$ Compound 1 (373 mg, $68 \%$ ) was isolated as a white solid. The data agreed with the reported data. ${ }^{1} \mathrm{H}$ NMR: $\delta=5.37$ (br. $\mathrm{d},{ }^{2} J=5.2 \mathrm{~Hz}, 1 \mathrm{H}, \mathrm{CH}=\mathrm{C}$ ), $4.19\left(\mathrm{~d},{ }^{4} \mathrm{~J}=2.4 \mathrm{~Hz}, 2 \mathrm{H}, \mathrm{OCH}_{2}\right), 3.47-3.31(\mathrm{~m}, 1 \mathrm{H}, \mathrm{OCH}), 2.42-$ $0.80(\mathrm{~m}, 41 \mathrm{H}), 0.68(\mathrm{~s}, 3 \mathrm{H}, \mathrm{Me}) \mathrm{ppm}$.

(3ß)-3-(Butyn-1-yloxy)cholest-5-ene (2): ${ }^{[10]}$ Compound 2 (353 mg, $62 \%$ ) was isolated as a white solid. The data agreed with the reported data. ${ }^{1} \mathrm{H}$ NMR: $\delta=5.35$ (br. $\mathrm{d},{ }^{2} J=5.2 \mathrm{~Hz}, 1 \mathrm{H}, \mathrm{CH}=\mathrm{C}$ ), $3.60\left(\mathrm{t},{ }^{3} \mathrm{~J}=7.1 \mathrm{~Hz}, 2 \mathrm{H}, \mathrm{OCH}_{2}\right), 3.19-3.03(\mathrm{~m}, 1 \mathrm{H}, \mathrm{OCH}), 2.45$ $\left(\mathrm{td},{ }^{3} \mathrm{~J}=2.6,7.1 \mathrm{~Hz}, 2 \mathrm{H}, \mathrm{OCH}_{2} \mathrm{CH}_{2}\right), 1.97\left(\mathrm{t},{ }^{4} \mathrm{~J}=2.6 \mathrm{~Hz}, 1 \mathrm{H}\right.$, $\left.\mathrm{CHCCH}_{2}\right), 1.88-0.80(\mathrm{~m}, 40 \mathrm{H}), 0.68$ (s, $\left.3 \mathrm{H}, \mathrm{Me}\right) \mathrm{ppm}$.

(3ß)-3-(5-Hexyn-1-yloxy)cholest-5-ene (3): Compound 3 (423 mg, $70 \%$ ) was isolated as a white solid. $R_{\mathrm{f}}=0.80$ (hexane/EtOAc, 9:1), m.p. $112-114{ }^{\circ} \mathrm{C} .{ }^{1} \mathrm{H}$ NMR: $\delta=5.34$ (br. d, ${ }^{3} J=5.2 \mathrm{~Hz}, 1 \mathrm{H}$, $\mathrm{CH}=\mathrm{C}), 3.47\left(\mathrm{t},{ }^{3} \mathrm{~J}=7.1 \mathrm{~Hz}, 2 \mathrm{H}, \mathrm{OCH}_{2}\right), 3.19-3.05(\mathrm{~m}, 1 \mathrm{H}$, $\mathrm{OCH}), 2.45\left(\mathrm{td},{ }^{3} \mathrm{~J}=7.1,2.6 \mathrm{~Hz}, 2 \mathrm{H}, \mathrm{OCH}_{2} \mathrm{CH}_{2}\right), 1.97\left(\mathrm{t},{ }^{4} \mathrm{~J}=\right.$ $\left.2.6 \mathrm{~Hz}, 1 \mathrm{H}, \mathrm{CHCCH}{ }_{2}\right), 1.88-0.80(\mathrm{~m}, 40 \mathrm{H}), 0.68(\mathrm{~s}, 3 \mathrm{H}, \mathrm{Me})$ ppm. ${ }^{13} \mathrm{C}$ NMR: $\delta=141.1(C=\mathrm{CH}), 121.5(\mathrm{C}=\mathrm{CH}), 84.4(\mathrm{CH} \equiv C)$, $79.0(\mathrm{OCH}), 68.3\left(\mathrm{OCH}_{2}\right), 67.4(\mathrm{CH} \equiv \mathrm{C}), 56.8,56.2,50.2,42.3$, $39.8,39.5,39.2,37.3,36.9,36.2,35.8,32.0,31.9,29.2,28.5,28.3$, $28.0,25.3,24.3,23.8,22.8,19.4,18.8,18.3,11.9$ ppm. HRMS (ESI): calcd. for $\mathrm{C}_{33} \mathrm{H}_{55} \mathrm{O}[\mathrm{M}+\mathrm{H}]^{+}$467.4247; found 467.4262 (3.2 ppm).

General Procedures for the Cycloaddition Reaction to Alkyne Cholesterol Ethers

Method 1: The oxime ( 2 equiv.), Ch-T ( 2.5 equiv.), and EtOH ( $1 \mathrm{~mL}$ per $0.1 \mathrm{mmol}$ oxime) were put into a round-bottomed flask, and alkyne 1, 2, or 3 (1 equiv.) was added. The mixture was stirred at room temp. To overcome solubility issues with pyrene oxime, the mixture was sonicated for $10 \mathrm{~min}$ before the reaction. The mixture was stirred for $1 \mathrm{~h}$, then the solvent was removed. The residue was dissolved in chloroform, and this solution was washed with $\mathrm{H}_{2} \mathrm{O}$. The organic layer was dried with anhydrous $\mathrm{Na}_{2} \mathrm{SO}_{4}$, and the solvent was removed. The crude reaction products were purified by flash column chromatography (hexane/EtOAc 0-5\%).

Method 2 (MW Activation): The oxime (2 equiv.), Ch-T (2.5 equiv.), and $\mathrm{EtOH}(3 \mathrm{~mL}$ per $0.5 \mathrm{mmol}$ oxime) were put into a $10 \mathrm{~mL}$ microwave vessel, and cholesteryl propargyl ether 1 (1 equiv.) was added. The mixture was heated $\left(100^{\circ} \mathrm{C}\right.$ for $1 \mathrm{~h}$ with benzaldehyde oxime; $60^{\circ} \mathrm{C}$ for $30 \mathrm{~min}$ for 1-naphthaldehyde or 1-pyrene oxime) in a microwave reactor $\left(P_{\max }=300 \mathrm{~W}\right)$. The solvent was removed, and the residue was dissolved in chloroform and washed with $\mathrm{H}_{2} \mathrm{O}$. The organic layer was dried with anhydrous $\mathrm{Na}_{2} \mathrm{SO}_{4}$, and the solvent was removed under reduced pressure. The crude reaction products were purified by flash column chromatography (hexane/EtOAc $0-5 \%)$.

Method 3: The oxime (2 equiv.) was put into a round-bottomed flask, and Ch-T (2.5 equiv.) in EtOH (25\% aq.; $1 \mathrm{~mL}$ per $0.1 \mathrm{mmol}$ oxime) was added. The resulting solution was allowed to stir for 10 min at room temp., after which the alkyne (1 equiv.) was added. The mixture was stirred for $1 \mathrm{~h}$, then the solvent was removed. The residue was dissolved in chloroform and washed with $\mathrm{H}_{2} \mathrm{O}$. The organic layer was dried with anhydrous $\mathrm{Na}_{2} \mathrm{SO}_{4}$, and the solvent was removed. The crude reaction products were purified by flash column chromatography (petroleum ether/EtOAc 0-10\%).

5-I(Cholest-5-en-3ß-yloxy)methyl]-3-(phenyl)isoxazole (4a): Compound 4a (Method 1: $25 \mathrm{mg}, 19 \%$; Method 2: $103 \mathrm{mg}, 78 \%$ ) was isolated as a white solid. $R_{\mathrm{f}}=0.54$ (hexane/EtOAc, 9:1), m.p. 123$125^{\circ} \mathrm{C} .{ }^{1} \mathrm{H}$ NMR: $\delta=7.85-7.76(\mathrm{~m}, 2 \mathrm{H}, \mathrm{Ar}-\mathrm{H}), 7.48-7.40(\mathrm{~m}, 3$ $\mathrm{H}, \mathrm{Ar}-\mathrm{H}), 6.56$ (s, $1 \mathrm{H}$, isoxazole-H), 5.37 (br. s, $1 \mathrm{H}, \mathrm{CH}=\mathrm{C}), 4.69$ (s, $\left.2 \mathrm{H}, \mathrm{OCH}_{2}\right), 3.42-3.27$ (m, 1 H, OCH), 2.47-0.79 (m, $\left.40 \mathrm{H}\right)$, $0.68(\mathrm{~s}, 3 \mathrm{H}, \mathrm{Me}) \mathrm{ppm} .{ }^{13} \mathrm{C} \mathrm{NMR}: \delta=170.7(\mathrm{C}=\mathrm{N}), 162.4$ (isoxazole-CO), $140.4(C=\mathrm{CH}), 130.0,129.1,128.9,126.8$ (Ar-C and 5 Ar- $\mathrm{CH}$ ), $122.1(\mathrm{C}=\mathrm{CH}), 100.7$ (isoxazole- $\mathrm{CH}), 79.6(\mathrm{OCH}), 61.1$ $\left(\mathrm{OCH}_{2}\right), 56.8,56.2,50.2,42.3,39.8,39.5,38.9,37.1,36.8,36.2$, $35.8,32.0,31.9,28.3,28.2,28.0,24.3,23.8,22.8,22.6,21.1,19.4$, 18.7, 11.9 ppm. HRMS (ESI): calcd. for $\mathrm{C}_{37} \mathrm{H}_{54} \mathrm{NO}_{2}[\mathrm{M}+\mathrm{H}]^{+}$ 544.4149; found 544.4165 (2.6 ppm).

5-[(2-Cholest-5-en-3ß-yloxy)ethyl]-3-(phenyl)isoxazole (5a): Following Method 1, compound 5a (57 mg, 44\%) was isolated as a white 
solid. $R_{\mathrm{f}}=0.60$ (hexane/EtOAc, 9:1), m.p. $112-114{ }^{\circ} \mathrm{C} .{ }^{1} \mathrm{H}$ NMR: $\delta=7.83-7.74$ (m, 2 H, Ar-H), 7.49-7.42 (m, 3 H, Ar-H), 6.39 (s, 1 $\mathrm{H}$, isoxazole-H), 5.35 (br. s, $1 \mathrm{H}, \mathrm{CH}=\mathrm{C}), 3.86\left(\mathrm{t},{ }^{3} J=6.6 \mathrm{~Hz}, 2\right.$ $\left.\mathrm{H}, \mathrm{OCH}_{2}\right), 3.31-3.08(\mathrm{~m}, 1 \mathrm{H}, \mathrm{OCH}), 3.11\left(\mathrm{t},{ }^{3} \mathrm{~J}=6.6 \mathrm{~Hz}, 2 \mathrm{H}\right.$, isoxazole- $\left.\mathrm{CH}_{2}\right), 2.45-0.80(\mathrm{~m}, 40 \mathrm{H}), 0.67(\mathrm{~s}, 3 \mathrm{H}, \mathrm{Me}) \mathrm{ppm} .{ }^{13} \mathrm{C}$ NMR: $\delta=170.6(\mathrm{C}=\mathrm{N}), 162.2$ (isoxazole-CO), $140.1(C=\mathrm{CH})$, 130.0, 129.2, 128.8, 126.9 (Ar- $\mathrm{C}$ and $5 \mathrm{Ar}-\mathrm{CH}), 122.0(\mathrm{C}=\mathrm{CH})$, 103.4 (isoxazole- $\mathrm{CH}), 79.6(\mathrm{OCH}), 65.0\left(\mathrm{OCH}_{2}\right), 56.8,56.2,50.2$, 42.4, 39.8, 39.5, 39.2, 37.2, 36.9, 36.2, 35.8, 32.1, 31.9, 28.4, 28.2, 28.0, 24.3, 23.8, 22.8, 22.6, 21.1, 19.4, 18.8, 11.9 ppm. HRMS (ESI): calcd. for $\mathrm{C}_{38} \mathrm{H}_{56} \mathrm{NO}_{2}[\mathrm{M}+\mathrm{H}]^{+}$558.4306; found 558.4306 (0 ppm).

5-[(4-Cholest-5-en-3ß-yloxy)-1-butyl]-3-(phenyl)isoxazole (6a): Following Method 1, compound 6a (115 mg, 90\%) was isolated as a white solid. $R_{\mathrm{f}}=0.60$ (hexane/EtOAc, 9:1), m.p. $128-130{ }^{\circ} \mathrm{C} .{ }^{1} \mathrm{H}$ NMR: $\delta=7.83-7.74(\mathrm{~m}, 2 \mathrm{H}, \mathrm{Ar}-\mathrm{H}), 7.49-7.42(\mathrm{~m}, 3 \mathrm{H}, \mathrm{Ar}-\mathrm{H})$, $6.32(\mathrm{~s}, 1 \mathrm{H}$, isoxazole-H), 5.35 (br. s, $1 \mathrm{H}, \mathrm{CH}=\mathrm{C}), 3.53\left(\mathrm{t},{ }^{3} \mathrm{~J}=\right.$ $\left.6.3 \mathrm{~Hz}, 2 \mathrm{H}, \mathrm{OCH}_{2}\right), 3.27-3.08(\mathrm{~m}, 1 \mathrm{H}, \mathrm{OCH}), 2.90\left(\mathrm{t},{ }^{3} \mathrm{~J}=6.3 \mathrm{~Hz}\right.$, $2 \mathrm{H}$, isoxazole- $\left.\mathrm{CH}_{2}\right), 2.44-0.80(\mathrm{~m}, 44 \mathrm{H}), 0.67(\mathrm{~s}, 3 \mathrm{H}, \mathrm{Me}) \mathrm{ppm}$. ${ }^{13} \mathrm{C} \mathrm{NMR}: \delta=174.0(\mathrm{C}=\mathrm{N}), 162.4$ (isoxazole-CO), $141.0(C=\mathrm{CH})$, 129.9, 129.4, 128.9, 126.8 (Ar-C and $5 \mathrm{Ar}-\mathrm{CH}), 121.6(\mathrm{C}=\mathrm{CH}), 99.0$ (isoxazole-CH), $79.1(\mathrm{OCH}), 67.3\left(\mathrm{OCH}_{2}\right), 56.8,56.2,50.2,42.3$, $39.8,39.5,39.2,38.7,37.3,36.9,36.2,31.9,31.0,30.3,29.6,28.9$, 28.5, 26.7, 24.4, 24.3, 23.8, 22.8, 22.6, 21.1, 18.8, 11.9 ppm. HRMS (ESI): calcd. for $\mathrm{C}_{40} \mathrm{H}_{60} \mathrm{NO}_{2}[\mathrm{M}+\mathrm{H}]^{+}$586.4619; found 586.4651 (5.5 ppm).

\section{5-[(Cholest-5-en-3及-yloxy)methyl]-3-(1-naphthyl)isoxazole}

(4b): Compound 4b (Method 1: $61 \mathrm{mg}, 43 \%$; Method 2: $138 \mathrm{mg}, 96 \%$ ) was isolated as a white solid. $R_{\mathrm{f}}=0.62$ (hexane/EtOAc, 9:1), m.p. 146-148 ${ }^{\circ} \mathrm{C} .{ }^{1} \mathrm{H}$ NMR: $\delta=8.42-8.33(\mathrm{~m}, 1 \mathrm{H}, \mathrm{Ar}-\mathrm{H}), 7.98-7.85$ (m, 2 H, Ar-H), 7.73-7.65 (m, 1 H, Ar-H), 7.53-7.47 (m, 3 H, Ar$\mathrm{H}), 6.57$ (s, $1 \mathrm{H}$, isoxazole-H), 5.38 (br. s, $1 \mathrm{H}, \mathrm{CH}=\mathrm{C}), 4.76$ (s, 2 $\left.\mathrm{H}, \mathrm{OCH}_{2}\right), 3.48-3.32(\mathrm{~m}, 1 \mathrm{H}, \mathrm{OCH}), 2.53-2.24(\mathrm{~m}, 2 \mathrm{H}), 2.10$ $1.74(\mathrm{~m}, 6 \mathrm{H}), 1.66-0.80(\mathrm{~m}, 32 \mathrm{H}), 0.68(\mathrm{~s}, 3 \mathrm{H}, \mathrm{Me}) \mathrm{ppm} .{ }^{13} \mathrm{C}$ NMR: $\delta=170.0(\mathrm{C}=\mathrm{N}), 162.5$ (isoxazole-CO), $140.4(C=\mathrm{CH})$, 133.9, 131.0, 130.2, 128.5, 127.8, 127.0, 126.9, 126.3, 125.7, 125.2 (3 Ar-C and $7 \mathrm{Ar}-\mathrm{CH}), 122.2(\mathrm{C}=\mathrm{CH}), 104.1$ (isoxazole- $\mathrm{CH}), 79.9$ $(\mathrm{OCH}), 61.2\left(\mathrm{OCH}_{2}\right), 56.8,56.2,50.2,42.3,39.8,39.6,39.0,37.2$, $36.9,36.2,35.8,32.0,31.9,28.3,28.2,28.0,24.3,23.8,22.8,22.6$, $21.1,19.4,18.8,11.9$ ppm. HRMS (ESI): calcd. for $\mathrm{C}_{41} \mathrm{H}_{56} \mathrm{NO}_{2}[\mathrm{M}$ $+\mathrm{H}]^{+}$594.4306; found 594.4319 (2.2 ppm).

5-[(2-Cholest-5-en-3ß-yloxy)ethyl]-3-(1-naphthyl)isoxazole (5b): Following Method 1, compound $\mathbf{5 b}(76 \mathrm{mg}, 54 \%)$ was isolated as a white solid. $R_{\mathrm{f}}=0.65$ (hexane/EtOAc, 9:1), m.p. $134-136{ }^{\circ} \mathrm{C} .{ }^{1} \mathrm{H}$ NMR: $\delta=8.41-8.34(\mathrm{~m}, 1 \mathrm{H}, \mathrm{Ar}-\mathrm{H}), 7.99-7.87(\mathrm{~m}, 2 \mathrm{H}, \mathrm{Ar}-\mathrm{H})$, 7.73-7.65 (m, $1 \mathrm{H}$, Ar-H), 7.57-7.46 (m, 3 H, Ar-H), 6.42 (s, $1 \mathrm{H}$, isoxazole-H), 5.35 (br. s, $1 \mathrm{H}, \mathrm{CH}=\mathrm{C}), 3.87\left(\mathrm{t},{ }^{3} \mathrm{~J}=6.6 \mathrm{~Hz}, 2 \mathrm{H}\right.$, $\left.\mathrm{OCH}_{2}\right), 3.30-3.10(\mathrm{~m}, 1 \mathrm{H}, \mathrm{OCH}), 3.14\left(\mathrm{t},{ }^{3} \mathrm{~J}=6.6 \mathrm{~Hz}, 2 \mathrm{H}\right.$, isoxazole- $\left.\mathrm{CH}_{2}\right), 2.45-2.14(\mathrm{~m}, 2 \mathrm{H}), 2.10-0.80(\mathrm{~m}, 38 \mathrm{H}), 0.67(\mathrm{~s}, 3 \mathrm{H}$, Me) ppm. ${ }^{13} \mathrm{C}$ NMR: $\delta=170.8(\mathrm{C}=\mathrm{N}), 162.6$ (isoxazole-CO), 140.7 $(C=\mathrm{CH}), 133.8,131.1,130.0,128.4,127.6,127.3,126.9,126.2$, 125.7, 125.2 (3 Ar-C and $7 \mathrm{Ar}-\mathrm{CH}), 121.9(\mathrm{C}=\mathrm{CH}), 103.4$ (isoxazole-CH), $79.5(\mathrm{OCH}), 65.1\left(\mathrm{OCH}_{2}\right), 56.8,56.2,50.2,42.3,39.8$, $39.5,39.1,37.2,36.9,36.2,35.8,32.0,31.9,28.4,28.2,28.0,24.3$, 23.8, 22.8, 22.6, 21.1, 19.4, 18.7, 11.9 ppm. HRMS (ESI): calcd. for $\mathrm{C}_{42} \mathrm{H}_{58} \mathrm{NO}_{2}[\mathrm{M}+\mathrm{H}]^{+}$608.4462; found 608.4487 (4.1 ppm).

5-[(4-Cholest-5-en-3ß-yloxy)-1-butyl]-3-(1-naphthyl)isoxazole (6b): Following Method 1, compound $\mathbf{6 b}(125 \mathrm{mg}, 90 \%)$ was isolated as a white solid. $R_{\mathrm{f}}=0.69$ (hexane/EtOAc, 9:1), m.p. $130-132{ }^{\circ} \mathrm{C} .{ }^{1} \mathrm{H}$ NMR: $\delta=8.41-8.33(\mathrm{~m}, 1 \mathrm{H}, \mathrm{Ar}-\mathrm{H}), 7.98-7.87(\mathrm{~m}, 2 \mathrm{H}, \mathrm{Ar}-\mathrm{H})$, 7.72-7.66 (m, 1 H, Ar-H), 7.59-7.49 (m, 3 H, Ar-H), 6.32 (s, $1 \mathrm{H}$, isoxazole- $\mathrm{H}$ ), 5.35 (br. s, $1 \mathrm{H}, \mathrm{CH}=\mathrm{C}), 3.53\left(\mathrm{t},{ }^{3} \mathrm{~J}=6.3 \mathrm{~Hz}, 2 \mathrm{H}\right.$,
$\left.\mathrm{OCH}_{2}\right), 3.27-3.08(\mathrm{~m}, 1 \mathrm{H}, \mathrm{OCH}), 2.90\left(\mathrm{t},{ }^{3} \mathrm{~J}=6.3 \mathrm{~Hz}, 2 \mathrm{H}\right.$, isoxazole- $\left.\mathrm{CH}_{2}\right), 2.44-0.80(\mathrm{~m}, 44 \mathrm{H}), 0.67(\mathrm{~s}, 3 \mathrm{H}, \mathrm{Me}) \mathrm{ppm} .{ }^{13} \mathrm{C} \mathrm{NMR}$ : $\delta=172.2(\mathrm{C}=\mathrm{N}), 161.4$ (isoxazole-CO), $139.9(C=\mathrm{CH}), 132.7$, 130.0, 129.0, 127.4, 126.6, 126.2, 125.9, 125.2, 124.7, 124.1 (3 Ar$\mathrm{C}$ and $7 \mathrm{Ar}-\mathrm{CH}), 120.5(\mathrm{C}=\mathrm{CH}), 101.4$ (isoxazole- $\mathrm{CH}$ ), 78.1 $(\mathrm{OCH}), 66.3\left(\mathrm{OCH}_{2}\right), 55.7,55.1,49.1,41.3,38.7,38.5,38.1,36.2$, $35.8,35.2,34.8,30.9,30.8,28.6,27.4,27.2,27.0,25.6,23.4,23.3$, $22.8,21.5,20.0,18.4,17.7,10.8 \mathrm{ppm}$. HRMS (ESI): calcd. for $\mathrm{C}_{44} \mathrm{H}_{62} \mathrm{NO}_{2}[\mathrm{M}+\mathrm{H}]^{+}$636.4775; found 636.4812 (5.8 ppm).

5-[(Cholest-5-en-3及-yloxy)methyl]-3-(1-pyrenyl)isoxazole (4c): Compound 4c (Method 1: $27 \mathrm{mg}, 17 \%$; Method 2: $113 \mathrm{mg}, 71 \%$ ) was isolated as a white solid. $R_{\mathrm{f}}=0.68$ (hexane/EtOAc, 9:1), m.p. 162 $164{ }^{\circ} \mathrm{C} .{ }^{1} \mathrm{H}$ NMR: $\delta=8.57\left(\mathrm{~d},{ }^{3} J=9.3 \mathrm{~Hz}, 1 \mathrm{H}, \mathrm{Ar}-\mathrm{H}\right), 8.36\left(\mathrm{~d},{ }^{3} J\right.$ $=8.1 \mathrm{~Hz}, 1 \mathrm{H}, \mathrm{Ar}-\mathrm{H}), 8.27-7.98(\mathrm{~m}, 7 \mathrm{H}, \mathrm{Ar}-\mathrm{H}), 6.59(\mathrm{~s}, 1 \mathrm{H}$, isoxazole-H), 5.34 (br. s, $1 \mathrm{H}, \mathrm{CH}=\mathrm{C}), 4.63\left(\mathrm{~s}, 2 \mathrm{H}, \mathrm{OCH}_{2}\right), 3.44$ 3.27 (m, $1 \mathrm{H}, \mathrm{OCH}), 2.53-0.80(\mathrm{~m}, 40 \mathrm{H}), 0.67$ (s, $3 \mathrm{H}, \mathrm{Me}) \mathrm{ppm}$. ${ }^{13} \mathrm{C}$ NMR: $\delta=170.0(\mathrm{C}=\mathrm{N}), 162.5$ (isoxazole-CO), $140.7(C=\mathrm{CH})$, $132.5,131.3,130.6,128.7,128.4,126.2,125.9,125.6,125.1,125.0$, 124.9, $122.7(7 \mathrm{Ar}-\mathrm{C}$ and $9 \mathrm{Ar}-\mathrm{CH}), 121.8(\mathrm{C}=\mathrm{CH}), 104.3$ (isoxazole- $\mathrm{CH}), 82.0(\mathrm{OCH}), 62.0\left(\mathrm{OCH}_{2}\right), 56.8,56.2,50.9,50.2,42.3$, $42.3,39.8,39.5,37.2,36.5,36.2,35.8,31.9,31.6,28.2,28.0,24.2$, 23.8, 22.8, 22.6, 21.1, 19.4, 18.8, 11.9 ppm. HRMS (ESI): calcd. for $\mathrm{C}_{47} \mathrm{H}_{58} \mathrm{NO}_{2}[\mathrm{M}+\mathrm{H}]^{+}$668.4462; found 668.4437 (-3.7 ppm).

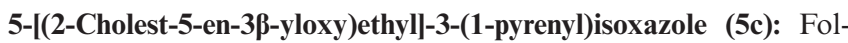
lowing Method 1 , compound $\mathbf{5 c}(57 \mathrm{mg}, 35 \%)$ was isolated as a white solid. $R_{\mathrm{f}}=0.70$ (hexane/EtOAc, 9:1), m.p. $160-162{ }^{\circ} \mathrm{C} .{ }^{1} \mathrm{H}$ NMR: $\delta=8.57\left(\mathrm{~d},{ }^{3} J=9.3 \mathrm{~Hz}, 1 \mathrm{H}, \mathrm{Ar}-\mathrm{H}\right), 8.32\left(\mathrm{~d},{ }^{3} J=8.1 \mathrm{~Hz}\right.$, $1 \mathrm{H}, \mathrm{Ar}-\mathrm{H}), 8.20-7.93(\mathrm{~m}, 7 \mathrm{H}, \mathrm{Ar}-\mathrm{H}), 6.44(\mathrm{~s}, 1 \mathrm{H}$, isoxazole-H), 5.37 (br. s, $1 \mathrm{H}, \mathrm{CH}=\mathrm{C}), 3.79\left(\mathrm{t},{ }^{3} J=6.7 \mathrm{~Hz}, 2 \mathrm{H}, \mathrm{OCH}_{2}\right), 3.32$ $3.09\left(\mathrm{~m}, 3 \mathrm{H}, \mathrm{OCH}\right.$, isoxazole- $\left.\mathrm{CH}_{2}\right), 2.48-0.80(\mathrm{~m}, 40 \mathrm{H}), 0.67(\mathrm{~s}$, $3 \mathrm{H}, \mathrm{Me}$ ) ppm. ${ }^{13} \mathrm{C}$ NMR: $\delta=170.6(\mathrm{C}=\mathrm{N}), 162.6$ (isoxazole-CO), $140.7(C=\mathrm{CH}), 132.4,131.3,130.5,128.6,128.4,126.2,125.9$, 125.7, 125.1, 125.0, 124.9, 122.7 (7 Ar-C and 9 Ar-CH), 121.7 $(\mathrm{C}=\mathrm{CH}), 103.3$ (isoxazole- $\mathrm{CH}), 81.9(\mathrm{OCH}), 65.1\left(\mathrm{OCH}_{2}\right), 56.8$, $56.2,50.3,50.2,42.3,42.1,39.8,39.5,37.2,36.4,36.2,35.8,32.0$, $31.9,28.3,28.0,24.3,23.8,22.8,22.6,21.1,19.4,18.7,11.9$ ppm. HRMS (ESI): calcd. for $\mathrm{C}_{48} \mathrm{H}_{60} \mathrm{NO}_{2}[\mathrm{M}+\mathrm{H}]^{+}$682.4619; found $682.4638(2.8 \mathrm{ppm})$.

5-[(4-Cholest-5-en-3ß-yloxy)-1-butyl]-3-(1-pyrenyl)isoxazole (6c): Following Method 1, compound $\mathbf{6 c}(135 \mathrm{mg}, 80 \%)$ was isolated as a white solid. $R_{\mathrm{f}}=0.79$ (hexane/EtOAc, 9:1), m.p. $155-157^{\circ} \mathrm{C} .{ }^{1} \mathrm{H}$ NMR: $\delta=8.57\left(\mathrm{~d},{ }^{3} J=9.3 \mathrm{~Hz}, 1 \mathrm{H}, \mathrm{Ar}-\mathrm{H}\right), 8.36\left(\mathrm{~d},{ }^{3} J=8.1 \mathrm{~Hz}\right.$, $1 \mathrm{H}, \mathrm{Ar}-\mathrm{H}), 8.24-7.98(\mathrm{~m}, 7 \mathrm{H}, \mathrm{Ar}-\mathrm{H}), 6.30(\mathrm{~s}, 1 \mathrm{H}$, isoxazole-H), 5.34 (br. s, $1 \mathrm{H}, \mathrm{CH}=\mathrm{C}), 3.55\left(\mathrm{t},{ }^{3} \mathrm{~J}=6.4 \mathrm{~Hz}, 2 \mathrm{H}, \mathrm{OCH}_{2}\right), 3.30$ $3.11(\mathrm{~m}, 1 \mathrm{H}, \mathrm{OCH}), 2.91\left(\mathrm{t},{ }^{3} \mathrm{~J}=6.4 \mathrm{~Hz}, 2 \mathrm{H}\right.$, isoxazole- $\left.\mathrm{CH}_{2}\right)$, 2.51-0.80 (m, $44 \mathrm{H}), 0.67$ (s, $3 \mathrm{H}, \mathrm{Me}) \mathrm{ppm} .{ }^{13} \mathrm{C} \mathrm{NMR:} \delta=172.7$ $(\mathrm{C}=\mathrm{N}), 161.9$ (isoxazole-CO), $140.8(C=\mathrm{CH}), 132.5,131.3,130.6$, 128.7, 128.4, 126.2, 125.9, 125.6, 125.1, 125.0, 124.9, 122.7 (7 Ar$\mathrm{C}$ and $9 \mathrm{Ar}-\mathrm{CH}$ ), $121.5(\mathrm{C}=\mathrm{CH}), 101.6$ (isoxazole- $\mathrm{CH}$ ), 79.9 $(\mathrm{OCH}), 66.4\left(\mathrm{OCH}_{2}\right), 56.4,55.3,49.1,41.3,38.7,38.5,38.1,36.2$, $35.8,35.2,34.8,30.9,30.8,28.6,27.4,27.2,27.0,25.6,23.4,23.3$, 22.8, 21.5, 21.1, 19.4, 18.8, 11.9 ppm. HRMS (ESI): calcd. for $\mathrm{C}_{50} \mathrm{H}_{64} \mathrm{NO}_{2}[\mathrm{M}+\mathrm{H}]^{+}$710.4932; found 710.4968 (5.1 ppm).

5-[(4-Cholest-5-en-3ß-yloxy)-1-butyl]-3-[6-(2H-1-benzopyran-2-one)isoxazole] (7): Following Method 1, compound 7 ( $26 \mathrm{mg}, 75 \%$ ) was isolated as a white solid. $R_{\mathrm{f}}=0.53$ (hexane/EtOAc, 8:2), m.p. 161$163{ }^{\circ} \mathrm{C} .{ }^{1} \mathrm{H}$ NMR: $\delta=8.15-8.00(\mathrm{~m}, 2 \mathrm{H}, \mathrm{Ar}-\mathrm{H}), 7.81\left(\mathrm{~d},{ }^{3} J=\right.$ $8.0 \mathrm{~Hz}, 1 \mathrm{H}, \mathrm{Ar}-\mathrm{H}), 7.49\left(\mathrm{~d},{ }^{3} J=7.6 \mathrm{~Hz}, 1 \mathrm{H}, \mathrm{Ar}-\mathrm{H}\right), 6.63(\mathrm{~s}, 1 \mathrm{H}$, isoxazole-H), $6.54\left(\mathrm{~d},{ }^{3} \mathrm{~J}=7.6 \mathrm{~Hz}, 1 \mathrm{H}, \mathrm{Ar}-\mathrm{H}\right), 5.35$ (br. s, $1 \mathrm{H}$, $\mathrm{CH}=\mathrm{C}), 3.54\left(\mathrm{t},{ }^{3} \mathrm{~J}=6.2 \mathrm{~Hz}, 2 \mathrm{H}, \mathrm{OCH}_{2}\right), 3.28-3.11(\mathrm{~m}, 1 \mathrm{H}$, $\mathrm{OCH}), 2.94\left(\mathrm{t},{ }^{3} \mathrm{~J}=6.2 \mathrm{~Hz}, 2 \mathrm{H}\right.$, isoxazole- $\left.\mathrm{CH}_{2}\right), 2.38-0.80(\mathrm{~m}, 44$ $\mathrm{H}), 0.68(\mathrm{~s}, 3 \mathrm{H}, \mathrm{Me}) \mathrm{ppm} .{ }^{13} \mathrm{C}$ NMR: $\delta=169.8(\mathrm{C}=\mathrm{N}), 163.8$ 
(isoxazole-CO), 160.2 (coumarin-CO), 159.2 (coumarin-CO$\mathrm{O} C=\mathrm{C}), 143.9$ (coumarin-COC $=C), 140.8(C=\mathrm{CH}), 133.5,132.5$, 130.1, 120.1, 119.0, 119.0 (2 Ar-C and 4 Ar-CH), $121.7(\mathrm{C}=C H)$, 101.6 (isoxazole- $\mathrm{CH}), 79.3(\mathrm{OCH}), 69.6\left(\mathrm{OCH}_{2}\right), 56.8,56.2,50.1$, $42.3,42.3,39.8,39.5,37.3,36.5,36.2,35.8,31.9,31.6,28.2,28.0$, $24.3,23.8,22.8,22.6,21.5,20.8,19.4,18.7,11.8$ ppm. HRMS (ESI): calcd. for $\mathrm{C}_{43} \mathrm{H}_{60} \mathrm{NO}_{4}[\mathrm{M}+\mathrm{H}]^{+} 654.4517$ found: 654.4535 (2.8 ppm).

5-[(4-Cholest-5-en-3ßyloxy)-1-butyl]-3-[4-(2-phenyldiazenyl)phenyl]isoxazole (8): Following Method 3, compound 8 (24 mg, 56\%) was isolated as a bright orange solid. $R_{\mathrm{f}}=0.42$ (petroleum ether/ EtOAc, 9:1), m.p. $103-105^{\circ} \mathrm{C} .{ }^{1} \mathrm{H}$ NMR: $\delta=8.05-7.91(\mathrm{~m}, 6 \mathrm{H}$, Ar-H), 7.58-7.45 (m, 3 H, Ar-H), $6.38(\mathrm{~s}, 1 \mathrm{H}$, isoxazole-H), 5.34 (br. d, $1 \mathrm{H}, \mathrm{CH}=\mathrm{C}), 3.51\left(\mathrm{t},{ }^{3} \mathrm{~J}=6.2 \mathrm{~Hz}, 2 \mathrm{H}, \mathrm{OCH}_{2}\right), 3.22-3.06$ $(\mathrm{m}, 1 \mathrm{H}, \mathrm{OCH}), 2.85\left(\mathrm{t},{ }^{3} \mathrm{~J}=7.4 \mathrm{~Hz}, 2 \mathrm{H}\right.$, isoxazole- $\left.\mathrm{CH}_{2}\right), 2.47-$ $0.77(\mathrm{~m}, 44 \mathrm{H}), 0.67(\mathrm{~s}, 3 \mathrm{H}, \mathrm{Me}) \mathrm{ppm} .{ }^{13} \mathrm{C} \mathrm{NMR}: \delta=174.4(\mathrm{C}=\mathrm{N})$, 153.3 (isoxazole-CO), $152.6(\mathrm{Ar}-\mathrm{C}), 141.0(\mathrm{C}=\mathrm{CH}), 131.7(\mathrm{Ar}-\mathrm{C})$, $131.3(\mathrm{Ar}-\mathrm{CH}), 129.2(\mathrm{Ar}-\mathrm{CH}), 128.9$ (Ar-C), 127.6 (Ar-CH), 127.2, $123.4(\mathrm{Ar}-\mathrm{CH}), 123.0(\mathrm{Ar}-\mathrm{CH}), 121.6(\mathrm{C}=\mathrm{CH}), 99.1$ (isoxazole-CH), $79.1(\mathrm{OCH}), 67.3\left(\mathrm{OCH}_{2}\right), 56.8,56.2,50.2,42.3$ (q-C), $39.8,39.5,39.2,37.3,36.9$ (q-C), 36.2, 35.8, 32.0, 31.9, 29.6, 28.5, 28.2, 28.0, 26.7 (isoxazole- $\mathrm{CH}_{2}$ ), 24.4, 24.3, 23.8, 22.8, 22.6, 21.1, 19.4, 18.7, 11.9 ppm. HRMS (ESI): calcd. for $\mathrm{C}_{46} \mathrm{H}_{64} \mathrm{~N}_{3} \mathrm{O}_{2}[\mathrm{M}+$ $\mathrm{H}]^{+}$690.4993; found 690.5018 (3.6 ppm).

$3 \beta-\left[N-(4-A m i n o b u t y l)\right.$ carbamoyl]cholesterol (9): ${ }^{[19]}$ In a modification of the literature procedure, a solution of cholesterol chloroformate $(2.25 \mathrm{~g}, 5 \mathrm{mmol})$ in dry toluene $(100 \mathrm{~mL})$ was added dropwise over $30 \mathrm{~min}$ to a solution of ethylenediamine $(16.7 \mathrm{~mL}$, $250 \mathrm{mmol})$ in dry toluene $(150 \mathrm{~mL})$ at $0{ }^{\circ} \mathrm{C}$ under a nitrogen atmosphere. The resulting solution was stirred at room temperature for $17 \mathrm{~h}$. The reaction mixture was washed with water, the organic extract was dried with anhydrous magnesium sulfate, and the solvents were evaporated to dryness in vacuo to give compound 9 (490 mg, $88 \%)$ as a white solid.

3及-( $N$-\{4-[(4-Formylbenzoyl)amino]butyl\} carbamoyl)cholesterol (11): DCC (99 mg, $0.48 \mathrm{mmol}, 1.2$ equiv.) and DMAP (59 mg, $0.48 \mathrm{mmol}, 1.2$ equiv.) were added to a suspension of aminocarbamate cholesterol 9 (200 mg, $0.40 \mathrm{mmol}$ ) and 4-(dimethoxymethyl)benzoic acid ${ }^{[20]}$ (94 mg, $0.48 \mathrm{mmol}, 1.2$ equiv.) in dry $\mathrm{CH}_{2} \mathrm{Cl}_{2}$ $(5 \mathrm{~mL})$ and dry toluene $(5 \mathrm{~mL})$ at $0{ }^{\circ} \mathrm{C}$ under argon. The mixture was stirred at room temperature for $17 \mathrm{~h}$, then the solvent was removed. The residue was purified by flash column chromatography (hexane/EtOAc 35\%) to give 3ß-cholest-5-en-3-yl $N$-\{3-[(4-dimethoxylbenzoyl)amino]butyl $\}$ carbamate (10) (144 mg, 53\%) as an offwhite solid. $R_{\mathrm{f}}=0.79$ (hexane/EtOAc, 7:3), m.p. $158-160{ }^{\circ} \mathrm{C} .{ }^{1} \mathrm{H}$ NMR: $\delta=7.78\left(\mathrm{~d},{ }^{3} J=8.3 \mathrm{~Hz}, 2 \mathrm{H}, \mathrm{Ar}-\mathrm{H}\right), 7.52\left(\mathrm{~d},{ }^{3} J=8.3 \mathrm{~Hz}\right.$, $2 \mathrm{H}, \mathrm{Ar}-\mathrm{H}), 6.38$ (br. s, $1 \mathrm{H}, \mathrm{NH}), 5.52$ [s, $\left.1 \mathrm{H}, \mathrm{CH}(\mathrm{OMe})_{2}\right], 5.43$ (br. s, $1 \mathrm{H}, \mathrm{CH}=\mathrm{C}$ ), 4.70 (br. s, $1 \mathrm{H}, \mathrm{NH}), 4.55-4.41(\mathrm{~m}, 1 \mathrm{H}$, $\mathrm{OCH}), 3.55-3.45$ (m, $2 \mathrm{H}, \mathrm{CONHCH}_{2}$ ), 3.32 (s, $\left.3 \mathrm{H}, \mathrm{OMe}\right), 3.31$ (s, $3 \mathrm{H}, \mathrm{OMe}$ ), 3.28-3.17 (m, $\left.2 \mathrm{H}, \mathrm{OCONHCH}_{2}\right), 2.41-0.96$ (m, 44 $\mathrm{H}), 0.68$ (s, $3 \mathrm{H}, \mathrm{Me}) \mathrm{ppm} .{ }^{13} \mathrm{C} \mathrm{NMR}: \delta=167.3(\mathrm{CONH}), 156.4$ $(\mathrm{OCONH}), 141.4(C=\mathrm{CH}), 139.8,134.7$ (2 Ar-C), 126.9, 126.9, 126.9, $126.8(4 \mathrm{Ar}-\mathrm{CH}), 122.5(\mathrm{C}=\mathrm{CH}), 102.4\left[\mathrm{CH}(\mathrm{OMe})_{2}\right], 76.6$ $(\mathrm{OCH}), 56.7,56.2,52.6,50.0,49.1,42.3,40.4,39.8,39.7,39.5,38.6$, $37.0,36.6,36.2,35.8,34.0,31.9,28.2,28.0,27.7,25.6,25.2,24.2$, 23.8, 22.8, 22.6, 21.1, 19.4, 18.8, 11.9 ppm. HRMS (ESI): calcd. for $\mathrm{C}_{42} \mathrm{H}_{67} \mathrm{~N}_{2} \mathrm{O}_{5}[\mathrm{M}+\mathrm{H}]^{+}$679.5044; found 679.5085 (6.0 ppm).

A solution of $\mathrm{HCl}(2 \mathrm{~N} ; 10 \mathrm{~mL})$ was added to a solution of crude protected aldehyde $\mathbf{1 0}(512 \mathrm{mg})$ in toluene $(10 \mathrm{~mL})$. After a few minutes in a sonication bath, the emulsion was stirred for $2 \mathrm{~h}$ at room temp. The reaction was carefully quenched with satd. aqueous $\mathrm{NaHCO}_{3}$ until the aqueous layer became neutral. The product was extracted with toluene. The organic phase was washed with water and dried with anhydrous $\mathrm{Na}_{2} \mathrm{SO}_{4}$, and the solvent was removed under reduced pressure. The residue was purified by flash column chromatography (hexane/EtOAc 30-60\%) to give compound 11 (50 mg, 10\% over the two steps from 9 to 11) as an offwhite solid. $R_{\mathrm{f}}=0.58$ (hexane/EtOAc, 8:2), m.p. $152-154{ }^{\circ} \mathrm{C} .{ }^{1} \mathrm{H}$ NMR: $\delta=10.08$ (s, $1 \mathrm{H}, \mathrm{CHO}$ ), 7.96 (s, $4 \mathrm{H}, \mathrm{Ar}-\mathrm{H}$ ), 6.70 (br. s, 1 $\mathrm{H}, \mathrm{NH}$ ), 5.37 (br. s, $1 \mathrm{H}, \mathrm{CH}=\mathrm{C}$ ), 4.76 (br. s, $1 \mathrm{H}, \mathrm{NH}$ ), 4.56-4.42 (m, $1 \mathrm{H}, \mathrm{OCH}), 3.58-3.48\left(\mathrm{~m}, 2 \mathrm{H}, \mathrm{CONHCH}_{2}\right), 3.28-3.17$ (m, 2 $\left.\mathrm{H}, \mathrm{OCONHCH}_{2}\right), 2.41-0.81(\mathrm{~m}, 44 \mathrm{H}), 0.67(\mathrm{~s}, 3 \mathrm{H}, \mathrm{Me}) \mathrm{ppm} .{ }^{13} \mathrm{C}$ NMR: $\delta=191.6(\mathrm{C}=\mathrm{O}), 167.8(\mathrm{CONH}), 157.4(\mathrm{OCONH}), 139.8$ $(C=\mathrm{CH}), 138.1,132.5$ (2 Ar-C), 130.9, 129.8, 128.8, 127.7 (4 Ar$\mathrm{CH}), 122.6(\mathrm{C}=\mathrm{CH}), 76.6(\mathrm{OCH}), 56.7,56.2,50.0,42.3,39.8,39.6$, $39.5,38.7,38.6,37.0,36.6,36.2,35.8,31.9,30.4,28.9,28.2,28.0$, 27.9, 24.3, 23.8, 23.0, 22.8, 22.6, 21.1, 19.3, 18.7, 11.0 ppm.

3及-( $N$-\{4-[(4-Hydroxyiminoformylbenzoyl)amino]butyl\}carbamoyl)cholesterol (12)

Method A - from Free Aldehyde 11: A mixture of aldehyde 11 (80 mg, $0.13 \mathrm{mmol})$, hydroxylamine hydrochloride $(14 \mathrm{mg}$, $0.19 \mathrm{mmol}, 1.5$ equiv.), and pyridine ( $15 \mathrm{mg}, 0.19 \mathrm{mmol}, 1.5$ equiv.) in $\mathrm{EtOH}(3 \mathrm{~mL})$ was heated to $125^{\circ} \mathrm{C}$ for $1 \mathrm{~h}$ in a microwave reactor $\left(10 \mathrm{~mL}\right.$ vessel, $\left.P_{\max }=300 \mathrm{~W}\right)$. The solvent was removed under reduced pressure. The residue was dissolved in chloroform, and this solution was washed with $\mathrm{H}_{2} \mathrm{O}$. The organic layer was dried with anhydrous $\mathrm{Na}_{2} \mathrm{SO}_{4}$, and the solvent was removed under reduced pressure to give oxime 12 (42 mg, 48\%) as an off-white solid.

Method B - from Protected Aldehyde 10: A mixture of $\mathbf{1 0}(80 \mathrm{mg}$, $0.12 \mathrm{mmol})$, hydroxylamine hydrochloride $(52 \mathrm{mg}, 0.71 \mathrm{mmol}$, 6 equiv.), and pyridine ( $28 \mathrm{mg}, 0.35 \mathrm{mmol}, 3$ equiv.) in $\mathrm{EtOH}$ $(3 \mathrm{~mL})$ was heated to $125^{\circ} \mathrm{C}$ for $1 \mathrm{~h}$ in a microwave reactor $(10 \mathrm{~mL}$ vessel, $\left.P_{\max }=300 \mathrm{~W}\right)$. The solvent was removed under reduced pressure. The residue was dissolved in chloroform, and this solution was washed with satd. aq. $\mathrm{NaHCO}_{3}$ and $\mathrm{H}_{2} \mathrm{O}$. The organic layer was dried with anhydrous $\mathrm{Na}_{2} \mathrm{SO}_{4}$, and the solvent was removed under reduced pressure to give pure oxime $12(75 \mathrm{mg}, 91 \%)$ as an off-white solid. $R_{\mathrm{f}}=0.60$ (hexane/EtOAc, 8:2), m.p. $156-158^{\circ} \mathrm{C}$. ${ }^{1} \mathrm{H}$ NMR: $\delta=8.15(\mathrm{~s}, 1 \mathrm{H}, \mathrm{CHNOH}), 7.80\left(\mathrm{~d},{ }^{3} \mathrm{~J}=8.1 \mathrm{~Hz}, 2 \mathrm{H}\right.$, Ar-H), 7.63 (d, $\left.{ }^{3} J=8.1 \mathrm{~Hz}, 2 \mathrm{H}, \mathrm{Ar}-\mathrm{H}\right), 6.47$ (br. s, $\left.1 \mathrm{H}, \mathrm{NH}\right), 5.36$ (br. s, $1 \mathrm{H}, \mathrm{CH}=\mathrm{C}), 4.73$ (br. s, $1 \mathrm{H}, \mathrm{NH}), 4.58-4.42(\mathrm{~m}, 1 \mathrm{H}$, $\mathrm{OCH}), 3.56-3.45\left(\mathrm{~m}, 2 \mathrm{H}, \mathrm{CONHCH}_{2}\right), 3.31-3.17(\mathrm{~m}, 2 \mathrm{H}$, $\left.\mathrm{OCONHCH}_{2}\right), 2.42-0.82(\mathrm{~m}, 44 \mathrm{H}), 0.68(\mathrm{~s}, 3 \mathrm{H}, \mathrm{Me}) \mathrm{ppm} .{ }^{13} \mathrm{C}$ NMR: $\delta=166.9(C O N H), 156.5(\mathrm{OCONH}), 149.9(\mathrm{C}=\mathrm{N}), 139.8$ $(C=\mathrm{CH}), 135.6,135.0$ (2 Ar-C), 127.4, 127.4, 127.1, 127.1 (4 Ar$\mathrm{CH}), 122.5(\mathrm{C}=\mathrm{CH}), 76.6(\mathrm{OCH}), 56.7,56.2,50.0,42.3,39.8,39.5$, $38.6,37.0,36.6,36.2,35.8,31.9,28.2,28.2,27.7,26.5,24.3,23.8$, $22.8,22.6,21.1,19.3,18.7,11.9$ ppm. IR: $\tilde{v}=3280,1765,1620$, 1461, 1405, 1382, 1211, 1041, 870, $692 \mathrm{~cm}^{-1}$. HRMS (ESI): calcd. for $\mathrm{C}_{40} \mathrm{H}_{62} \mathrm{~N}_{3} \mathrm{O}_{4}[\mathrm{M}+\mathrm{H}]^{+}$648.4735; found 648.4772 (5.7 ppm).

General Procedure for the Formation of Amidocarbamate-Linked Steroidal Conjugates 14a,b: The alkyne (1 equiv.), propargyl alcohol (leading to 14a), or phenyl propargyl ether (leading to $\mathbf{1 4 b}$ ) was added to a round-bottomed flask containing oxime $\mathbf{1 2}$ ( 2 equiv.) and Ch-T (2.5 equiv.) in EtOH ( $1 \mathrm{~mL}$ per $0.1 \mathrm{mmol}$ oxime). The mixture was stirred for $17 \mathrm{~h}$ at room temp. The mixture was extracted with toluene, and the organic phase was washed with $\mathrm{H}_{2} \mathrm{O}$. The organic layer was dried with anhydrous $\mathrm{Na}_{2} \mathrm{SO}_{4}$, and the solvent was removed under reduced pressure. The residue was purified by flash column chromatography (hexane/EtOAc 0-15\%) to give the title compounds.

$3 \beta-[N-(4-\{[4-(5-H y d r o x y m e t h y l) i s o x a z o l-3-y l] b e n z o y l a m i n o b u t y l\})-$ carbamoyl] cholesterol (14a): Compound 14a was isolated (11 mg, $21 \%$ ) as an off-white solid. $R_{\mathrm{f}}=0.26$ (hexane/EtOAc, 8:2), m.p. 
120-122 ${ }^{\circ} \mathrm{C} .{ }^{1} \mathrm{H}$ NMR: $\delta=7.84\left(\mathrm{~d},{ }^{3} J=8.0 \mathrm{~Hz}, 2 \mathrm{H}\right.$, Ar-H), 7.26 (d, $\left.{ }^{3} J=8.0 \mathrm{~Hz}, 2 \mathrm{H}, \mathrm{Ar}-\mathrm{H}\right), 6.58(\mathrm{~s}, 1 \mathrm{H}$, isoxazole-H), 5.39 (br. s, $1 \mathrm{H}, \mathrm{CH}=\mathrm{C}$ ), 4.81 (s, $2 \mathrm{H}, \mathrm{OCH}_{2}$ ), 4.74 (br. s, $1 \mathrm{H}, \mathrm{NH}$ ), 4.57-4.41 (m, $1 \mathrm{H}, \mathrm{OCH}), 3.63-3.55\left(\mathrm{~m}, 2 \mathrm{H}, \mathrm{CONHCH}_{2}\right), 3.26-3.11(\mathrm{~m}, 2$ $\mathrm{H}, \mathrm{OCONHCH} 2), 2.62-0.75(\mathrm{~m}, 44 \mathrm{H}), 0.67(\mathrm{~s}, 3 \mathrm{H}, \mathrm{Me}) \mathrm{ppm} .{ }^{13} \mathrm{C}$ NMR: $\delta=170.7(\mathrm{C}=\mathrm{N}), 166.9(C \mathrm{ONH}), 160.5$ (isoxazole-CO), $156.5(\mathrm{OCONH}), 140.4(\mathrm{C}=\mathrm{CH}), 134.6,134.1$ (2 Ar-C), 129.7, 128.7, 128.7, $128.1(4 \mathrm{Ar}-\mathrm{CH}), 122.3(\mathrm{C}=\mathrm{CH}), 99.8$ (isoxazole- $\mathrm{CH}$ ), $79.6(\mathrm{OCH}), 56.7,56.2,53.0,50.1,42.3,39.8,39.5,38.6,37.0,36.6$, $36.2,35.9,31.9,28.2,27.8,26.5,24.3,23.8,22.7,22.6,21.1,19.3$, 18.7, 11.9 ppm. IR: $\tilde{v}=3354,3293,1770,1632,1565,1480,1441$, $1375,1035,1021,890 \mathrm{~cm}^{-1}$. HRMS (ESI): calcd. for $\mathrm{C}_{43} \mathrm{H}_{64} \mathrm{~N}_{3} \mathrm{O}_{5}$ $[\mathrm{M}+\mathrm{H}]^{+}$702.4840; found 702.4867 (3.8 ppm).

$3 \beta-[N-(4-\{[4-(5-P h e n o x y m e t h y l) i s o x a z o l-3-y l] b e n z o y l a m i n o b u t y l\})-$ carbamoyl] cholesterol (14b): Compound 14b was isolated $(21 \mathrm{mg}$, $37 \%$ ) as an off-white solid. $R_{\mathrm{f}}=0.45$ (hexane/EtOAc, 8:2), m.p. 107-109 ${ }^{\circ} \mathrm{C}$. ${ }^{1} \mathrm{H}$ NMR: $\delta=7.88-7.71$ (m, $4 \mathrm{H}$, Ar-H), 7.50-7.41 $(\mathrm{m}, 3 \mathrm{H}, \mathrm{Ar}-\mathrm{H}), 7.25\left(\mathrm{~d},{ }^{3} \mathrm{~J}=8.0 \mathrm{~Hz}, 2 \mathrm{H}, \mathrm{Ar}-\mathrm{H}\right), 6.56(\mathrm{~s}, 1 \mathrm{H}$, isoxazole-H), 5.37 (br. s, $1 \mathrm{H}, \mathrm{CH}=\mathrm{C}$ ), 4.72 (br. s, $1 \mathrm{H}, \mathrm{NH}), 4.69$ (s, $\left.2 \mathrm{H}, \mathrm{OCH}_{2}\right), 4.55-4.40(\mathrm{~m}, 1 \mathrm{H}, \mathrm{OCH}), 3.65-3.55(\mathrm{~m}, 2 \mathrm{H}$, $\left.\mathrm{CONHCH}_{2}\right), 3.24-3.10\left(\mathrm{~m}, 2 \mathrm{H}, \mathrm{OCONHCH}_{2}\right), 2.60-0.77$ (m, 44 $\mathrm{H}), 0.68(\mathrm{~s}, 3 \mathrm{H}, \mathrm{Me}) \mathrm{ppm} .{ }^{13} \mathrm{C}$ NMR: $\delta=170.7(\mathrm{C}=\mathrm{N}), 167.0$ $(\mathrm{CONH}), 162.4$ (isoxazole-CO), $156.5(\mathrm{OCONH}), 140.5(C=\mathrm{CH})$, 134.6, 134.0, 130.0 (3 Ar-C), 129.8, 129.7, 129.6, 129.6, 128.7, 128.2, $127.8(9 \mathrm{Ar}-\mathrm{CH}), 122.2(\mathrm{C}=\mathrm{CH}), 100.7$ (isoxazole- $\mathrm{CH}), 79.7$ $(\mathrm{OCH}), 61.1\left(\mathrm{OCH}_{2}\right), 56.7,56.3,50.1,42.3,39.8,39.6,38.6,37.1$, $36.6,36.3,35.9,31.9,28.2,27.8,26.6,24.3,23.8,22.8,21.1,19.3$, 18.7, 11.9 ppm. IR: $\tilde{v}=3288,1768,1644,1570,1450,1383,1022$, $888 \mathrm{~cm}^{-1}$. HRMS (ESI): calcd. for $\mathrm{C}_{49} \mathrm{H}_{68} \mathrm{~N}_{3} \mathrm{O}_{5}[\mathrm{M}+\mathrm{H}]^{+} 778.5153$; found $778.5184(4.0 \mathrm{ppm})$

General Procedure for the Preparation of Aldehydes 15: A mixture of cholesterol (1.00 g, $2.59 \mathrm{mmol}), 2-$, 3-, or 4-(2-hydroxyethoxy)benzaldehyde (purchased from TCI Europe; $2.15 \mathrm{~g}, 12.93 \mathrm{mmol}$, 5 equiv.), and MK-10 (activated at $120^{\circ} \mathrm{C}$ overnight before use; $2.00 \mathrm{~g})$ in chloroform $(5 \mathrm{~mL})$ was heated to $90^{\circ} \mathrm{C}$ for $8 \mathrm{~h}$ in a microwave reactor $\left(10 \mathrm{~mL}\right.$ vessel, $\left.P_{\max }=300 \mathrm{~W}\right)$. The solvent was removed under reduced pressure, and hexane $(20 \mathrm{~mL})$ was added. The catalyst was removed by filtration and washed with hexane. The solvent was evaporated, and the residue was purified by flash column chromatography (hexane/EtOAc $0-10 \%$ ) to give the title compounds.

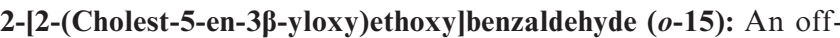
white solid $(485 \mathrm{mg}, 35 \%) . R_{\mathrm{f}}=0.62$ (hexane/EtOAc, 8:2), m.p. 86-88 ${ }^{\circ} \mathrm{C} .{ }^{1} \mathrm{H}$ NMR: $\delta=10.53$ (s, $\left.1 \mathrm{H}, \mathrm{CHO}\right), 7.88-7.80(\mathrm{~m}, 1 \mathrm{H}$, Ar-H), 7.59-7.47 (m, 1 H, Ar-H), 7.11-6.95 (m, 2 H, Ar-H), 5.41$5.29(\mathrm{~m}, 1 \mathrm{H}, \mathrm{CH}=\mathrm{C}), 4.23\left(\mathrm{t},{ }^{3} \mathrm{~J}=5.0 \mathrm{~Hz}, 2 \mathrm{H}, \mathrm{OCH}_{2}\right), 3.89\left(\mathrm{t},{ }^{3} \mathrm{~J}\right.$ $\left.=5.0 \mathrm{~Hz}, 2 \mathrm{H}, \mathrm{OCH}_{2}\right), 3.35-3.19(\mathrm{~m}, 1 \mathrm{H}, \mathrm{OCH}), 2.46-0.77(\mathrm{~m}, 40$ $\mathrm{H}), 0.68(\mathrm{~s}, 3 \mathrm{H}, \mathrm{Me})$ ppm. ${ }^{13} \mathrm{C}$ NMR: $\delta=189.9(\mathrm{C}=\mathrm{O}), 161.4$ (Ar-C), $140.6(C=\mathrm{CH}), 135.8,128.2(2 \mathrm{Ar}-\mathrm{H}), 125.1$ (Ar-C), 121.9 $(\mathrm{C}=\mathrm{CH}), 112.9(2 \mathrm{Ar}-\mathrm{H}), 79.9(\mathrm{OCH}), 76.6,68.5,65.9,56.8,56.2$, $50.2,42.3,39.8,39.5,39.1,37.2,36.9,36.2,35.8,31.9,28.4,28.2$, $28.0,24.3,23.8,22.8,22.6,21.1,19.4,18.7,11.9$ ppm. IR: $\tilde{v}=2765$, 1702, 1450, 1378, 1227, 1034, $760 \mathrm{~cm}^{-1}$. HRMS (ESI): calcd. for $\mathrm{C}_{36} \mathrm{H}_{55} \mathrm{O}_{3}[\mathrm{M}+\mathrm{H}]^{+}$535.4146; found 535.4131 (2.8 ppm).

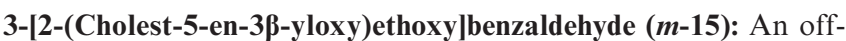
white solid (803 mg, 58\%). $R_{\mathrm{f}}=0.62$ (hexane/EtOAc, 8:2), m.p. 80 $82{ }^{\circ} \mathrm{C} .{ }^{1} \mathrm{H}$ NMR: $\delta=9.96(\mathrm{~s}, 1 \mathrm{H}, \mathrm{CHO}), 7.64-7.38$ (m, $3 \mathrm{H}, \mathrm{Ar}-$ $\mathrm{H}), 7.24-7.17(\mathrm{~m}, 1 \mathrm{H}, \mathrm{Ar}-\mathrm{H}), 5.49-5.32(\mathrm{~m}, 1 \mathrm{H}, \mathrm{CH}=\mathrm{C}), 4.17(\mathrm{t}$, $\left.{ }^{3} J=4.8 \mathrm{~Hz}, 2 \mathrm{H}, \mathrm{OCH}_{2}\right), 3.86\left(\mathrm{t},{ }^{3} J=4.8 \mathrm{~Hz}, 2 \mathrm{H}, \mathrm{OCH}_{2}\right), 3.35$ $3.19(\mathrm{~m}, 1 \mathrm{H}, \mathrm{OCH}), 2.57-0.80(\mathrm{~m}, 40 \mathrm{H}), 0.68$ (s, $3 \mathrm{H}, \mathrm{Me}) \mathrm{ppm}$. ${ }^{13} \mathrm{C}$ NMR: $\delta=191.1(\mathrm{C}=\mathrm{O}), 158.5(\mathrm{Ar}-\mathrm{C}), 139.8(C=\mathrm{CH}), 136.7$, 129.0 (2 Ar-H), 122.5 (Ar-C), $120.7(\mathrm{C}=\mathrm{CH}), 112.1$ (2 Ar-H), 78.8
(OCH) , 75.6, 67.0, 65.3, 55.8, 55.2, 49.2, 41.3, 38.8, 38.5, 38.0, 36.2, $35.9,35.2,34.77,30.94,30.9,27.4,27.2,27.0,23.3,22.8,21.8,21.6$, 20.1, 18.4, 17.7, 10.9 ppm. IR: $\tilde{v}=2753,1708,1460,1371,1232$, 1027, $691 \mathrm{~cm}^{-1}$. HRMS (ESI): calcd. for $\mathrm{C}_{36} \mathrm{H}_{55} \mathrm{O}_{3}[\mathrm{M}+\mathrm{H}]^{+}$ 535.4146 ; found 535.4135 (2.0 ppm)

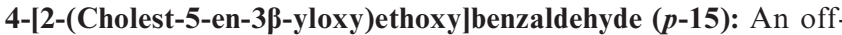
white solid (802 mg, 58\%). $R_{\mathrm{f}}=0.60$ (hexane/EtOAc, 8:2), m.p. 90-92 ${ }^{\circ} \mathrm{C} .{ }^{1} \mathrm{H}$ NMR: $\delta=9.88(\mathrm{~s}, 1 \mathrm{H}, \mathrm{CHO}), 7.84\left(\mathrm{~d},{ }^{3} J=8.7 \mathrm{~Hz}\right.$, $2 \mathrm{H}$, Ar-H), $7.03\left(\mathrm{~d},{ }^{3} J=8.7 \mathrm{~Hz}, 2 \mathrm{H}, \mathrm{Ar}-\mathrm{H}\right), 5.39-5.30(\mathrm{~m}, 1 \mathrm{H}$, $\mathrm{CH}=\mathrm{C}), 4.19\left(\mathrm{t},{ }^{3} J=4.9 \mathrm{~Hz}, 2 \mathrm{H}, \mathrm{OCH}_{2}\right), 3.86\left(\mathrm{t},{ }^{3} J=4.9 \mathrm{~Hz}, 2\right.$ $\left.\mathrm{H}, \mathrm{OCH}_{2}\right), 3.31-3.19(\mathrm{~m}, 1 \mathrm{H}, \mathrm{OCH}), 2.45-0.75(\mathrm{~m}, 40 \mathrm{H}), 0.67(\mathrm{~s}$, $3 \mathrm{H}, \mathrm{Me}) \mathrm{ppm} .{ }^{13} \mathrm{C}$ NMR: $\delta=190.8(\mathrm{C}=\mathrm{O}), 164.0$ (Ar-C), 140.7 $(C=\mathrm{CH}), 131.9,130.0(2 \mathrm{Ar}-\mathrm{H}), 121.8(\mathrm{Ar}-\mathrm{C}), 121.8(\mathrm{C}=\mathrm{CH}), 114.9$ (2 Ar-H), $79.9(\mathrm{OCH}), 76.6,68.1,66.2,65.9,56.8,56.2,50.2,42.3$, $39.8,39.5,39.0,37.2,36.9,36.2,35.8,32.0,31.9,31.6,28.4,24.3$, $23.8,22.8,22.7,22.6,21.1,19.4,18.7,11.9$ ppm. IR: $\tilde{v}=2761$, 1705, 1461, 1382, 1225, 1028, $832 \mathrm{~cm}^{-1}$. HRMS (ESI): calcd. for $\mathrm{C}_{36} \mathrm{H}_{55} \mathrm{O}_{3}[\mathrm{M}+\mathrm{H}]^{+}$535.4146; found 535.4168 (4.1 ppm).

General Procedure for the Preparation of Oximes 16: A mixture of aldehyde 15 (500 mg, $0.94 \mathrm{mmol})$, hydroxylamine hydrochloride (97 mg, $1.40 \mathrm{mmol}, 1.5$ equiv.), and pyridine (110 mg, $1.40 \mathrm{mmol}$, 1.5 equiv.) in $\mathrm{EtOH}$ was heated to $125^{\circ} \mathrm{C}$ for $1 \mathrm{~h}$ in a microwave reactor $\left(10 \mathrm{~mL}\right.$ vessel, $\left.P_{\max }=300 \mathrm{~W}\right)$. The solvent was removed under reduced pressure, the residue was dissolved in chloroform, and this solution was washed with $\mathrm{H}_{2} \mathrm{O}$. The organic layer was dried with anhydrous $\mathrm{Na}_{2} \mathrm{SO}_{4}$, and the solvent was removed to give the pure oximes as off-white solids in quantitative yields.

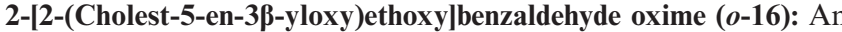
off-white solid (511 mg). $R_{\mathrm{f}}=0.57$ (hexane/EtOAc, 8:2), m.p. 112 $114{ }^{\circ} \mathrm{C} .{ }^{1} \mathrm{H}$ NMR: $\delta=8.55(\mathrm{~s}, 1 \mathrm{H}, \mathrm{CH}=\mathrm{NOH}), 8.20(\mathrm{~s}, 1 \mathrm{H}$, $\mathrm{CH}=\mathrm{NOH}), 7.72\left(\mathrm{~d},{ }^{3} \mathrm{~J}=7.8 \mathrm{~Hz}, 1 \mathrm{H}, \mathrm{Ar}-\mathrm{H}\right), 7.36-7.27(\mathrm{~m}, 2 \mathrm{H}$, Ar-H), 7.01-6.86 (m, $1 \mathrm{H}, \mathrm{Ar}-\mathrm{H}), 5.40-5.25$ (m, $1 \mathrm{H}, \mathrm{CH}=\mathrm{C}), 4.15$ $\left(\mathrm{t},{ }^{3} \mathrm{~J}=5.1 \mathrm{~Hz}, 2 \mathrm{H}, \mathrm{OCH}_{2}\right), 3.85\left(\mathrm{t},{ }^{3} J=5.0 \mathrm{~Hz}, 2 \mathrm{H}, \mathrm{OCH}_{2}\right)$, 3.35-3.20 (m, 1 H, OCH), 2.46-0.77 (m, $40 \mathrm{H}), 0.68(\mathrm{~s}, 3 \mathrm{H}, \mathrm{Me})$ ppm. ${ }^{13} \mathrm{C}$ NMR: $\delta=157.0($ Ar-C), $146.5(\mathrm{C}=\mathrm{N}), 140.8(C=\mathrm{CH})$, 131.1, $129.2(2 \mathrm{Ar}-\mathrm{H}), 123.4(\mathrm{Ar}-\mathrm{C}), 121.8(\mathrm{C}=\mathrm{CH}), 112.6(2 \mathrm{Ar}-$ H), $79.8(\mathrm{OCH}), 76.6,68.5,66.3,56.8,56.2,50.2,42.3,39.8,39.5$, $39.1,37.2,36.9,36.2,35.8,32.0,31.9,28.4,28.3,28.0,24.3,23.9$, $22.8,22.6,21.1,19.4,18.7,11.9$ ppm. IR: $\tilde{v}=3230,1452,1381$, 1225, 1029, $765 \mathrm{~cm}^{-1}$. HRMS (ESI): calcd. for $\mathrm{C}_{36} \mathrm{H}_{56} \mathrm{NO}_{3}[\mathrm{M}+$ $\mathrm{H}]^{+}$550.4255; found 550.4285 (5.4 ppm).

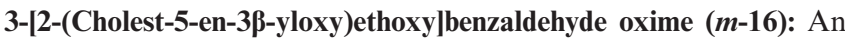
off-white solid (512 mg). $R_{\mathrm{f}}=0.59$ (hexane/EtOAc, 8:2), m.p. 110 $112{ }^{\circ} \mathrm{C} .{ }^{1} \mathrm{H}$ NMR: $\delta=8.08(\mathrm{~s}, 1 \mathrm{H}, \mathrm{CH}=\mathrm{NOH}), 7.35-7.07(\mathrm{~m}, 4$ $\mathrm{H}, \mathrm{Ar}-\mathrm{H}, \mathrm{CH}=\mathrm{NOH}$ ), 7.03-6.90 (m, $1 \mathrm{H}, \mathrm{Ar}-\mathrm{H}), 5.40-5.25$ (m, 1 $\mathrm{H}, \mathrm{CH}=\mathrm{C}), 4.13\left(\mathrm{t},{ }^{3} \mathrm{~J}=5.1 \mathrm{~Hz}, 2 \mathrm{H}, \mathrm{OCH}_{2}\right), 3.84\left(\mathrm{t},{ }^{3} J=5.1 \mathrm{~Hz}\right.$, $\left.2 \mathrm{H}, \mathrm{OCH}_{2}\right), 3.35-3.20(\mathrm{~m}, 1 \mathrm{H}, \mathrm{OCH}), 2.48-0.77(\mathrm{~m}, 40 \mathrm{H}), 0.68$ (s, $3 \mathrm{H}, \mathrm{Me}$ ) ppm. ${ }^{13} \mathrm{C}$ NMR: $\delta=158.1(\mathrm{Ar}-\mathrm{C}), 149.2(\mathrm{C}=\mathrm{N}), 139.8$ $(C=\mathrm{CH}), 132.4,128.7(2 \mathrm{Ar}-\mathrm{H}), 124.8(\mathrm{Ar}-\mathrm{C}), 120.7(\mathrm{C}=\mathrm{CH}), 115.9$ (2 Ar-H), $78.8(\mathrm{OCH}), 75.6,66.8,65.4,55.8,55.1,49.2,41.3,38.8$, $38.5,38.0,36.2,35.9,35.2,34.8,30.9,30.9,27.2,27.0,23.3,22.8$, $21.8,21.6,20.1,18.4,17.7,10.8$ ppm. IR: $\tilde{v}=3221,1465,1409$, 1384, 1227, 1025, $702 \mathrm{~cm}^{-1}$. HRMS (ESI): calcd. for $\mathrm{C}_{36} \mathrm{H}_{56} \mathrm{NO}_{3}$ $[\mathrm{M}+\mathrm{H}]^{+}$550.4255; found $550.4284(5.2 \mathrm{ppm})$.

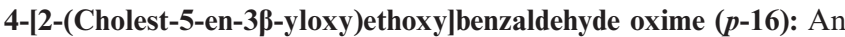
off-white solid (516 mg). $R_{\mathrm{f}}=0.60$ (hexane/EtOAc, 8:2), m.p. 120 $122{ }^{\circ} \mathrm{C} .{ }^{1} \mathrm{H}$ NMR: $\delta=8.07(\mathrm{~s}, 1 \mathrm{H}, \mathrm{CH}=\mathrm{NOH}), 7.49\left(\mathrm{~d},{ }^{3} J=\right.$ $9.0 \mathrm{~Hz}, 2 \mathrm{H}, \mathrm{Ar}-\mathrm{H}), 7.35$ (br. s, $1 \mathrm{H}, \mathrm{CH}=\mathrm{NOH}), 6.92\left(\mathrm{~d},{ }^{3} J=\right.$ $9.0 \mathrm{~Hz}, 2 \mathrm{H}, \mathrm{Ar}-\mathrm{H}), 5.42-5.29(\mathrm{~m}, 1 \mathrm{H}, \mathrm{CH}=\mathrm{C}), 4.13\left(\mathrm{t},{ }^{3} \mathrm{~J}=\right.$ $\left.5.1 \mathrm{~Hz}, 2 \mathrm{H}, \mathrm{OCH}_{2}\right), 3.84\left(\mathrm{t},{ }^{3} \mathrm{~J}=5.1 \mathrm{~Hz}, 2 \mathrm{H}, \mathrm{OCH}_{2}\right), 3.35-3.19$ (m, 1 H, OCH), 2.47-0.78 (m, $40 \mathrm{H}), 0.68(\mathrm{~s}, 3 \mathrm{H}, \mathrm{Me}) \mathrm{ppm} .{ }^{13} \mathrm{C}$ NMR: $\delta=160.4(\mathrm{Ar}-\mathrm{C}), 150.0(\mathrm{C}=\mathrm{N}), 140.8(C=\mathrm{CH}), 128.4(2 \mathrm{Ar}-$ 
H), $124.8(\mathrm{Ar}-\mathrm{C}), 121.7(\mathrm{C}=\mathrm{CH}), 115.4(2 \mathrm{Ar}-\mathrm{H}), 79.8(\mathrm{OCH}), 76.6$, $67.8,66.4,56.8,56.2,50.2,42.3,39.8,39.5,39.1,37.2,36.9,36.2$, $35.8,32.0,31.9,28.4,28.2,28.0,24.3,23.8,22.8,22.6,21.1,19.4$, 18.7, 11.9 ppm. IR: $\tilde{v}=3222,1431,1379,1228,1024,842 \mathrm{~cm}^{-1}$. HRMS (ESI): calcd. for $\mathrm{C}_{36} \mathrm{H}_{56} \mathrm{NO}_{3}[\mathrm{M}+\mathrm{H}]^{+}$550.4255; found $550.4272(3.1 \mathrm{ppm})$.

General Procedure for Cycloaddition Between (Prop-2-yn-1-yloxy)benzene and Putative Nitrile Oxides 17, derived from 16: (Prop-2yn-1-yloxy)benzene (16 $\mathrm{mg}, 0.12 \mathrm{mmol}$ ) was put into a round-bottomed flask, a solution of oxime $\mathbf{1 6}(100 \mathrm{mg}, 0.18 \mathrm{mmol}, 1.5$ equiv.) and Ch-T (54 mg, $0.24 \mathrm{mmol}, 2$ equiv.) in EtOH ( $1 \mathrm{~mL}$ per $0.1 \mathrm{mmol}$ oxime) was added. The mixture was stirred for $17 \mathrm{~h}$ at room temp. The solvent was removed under reduced pressure, the residue was dissolved in toluene, and this solution was washed with $\mathrm{H}_{2} \mathrm{O}$. The organic layer was dried with anhydrous $\mathrm{Na}_{2} \mathrm{SO}_{4}$, and the solvent was removed under reduced pressure. The crude reaction product was purified by flash column chromatography (hexane/ EtOAc $0-10 \%$ ) to give the title compounds as off-white solids.

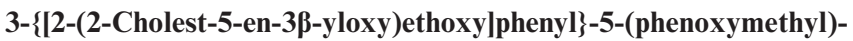
isoxazole (o-18): Compound $\boldsymbol{o}-18(20 \mathrm{mg}, 25 \%)$ was isolated. $R_{\mathrm{f}}=$ 0.40 (hexane/EtOAc, 8:2). ${ }^{1} \mathrm{H}$ NMR: $\delta=7.72\left(\mathrm{~d},{ }^{3} \mathrm{~J}=7.9 \mathrm{~Hz}, 1 \mathrm{H}\right.$, Ar-H), 7.38-7.20 (m, 3 H, Ar-H), 7.09-6.84 (m, 5 H, Ar-H), 6.57 (s, $1 \mathrm{H}$, isoxazole-H), 5.35 (br. s, $1 \mathrm{H}, \mathrm{CH}=\mathrm{C}), 5.18\left(\mathrm{~s}, 2 \mathrm{H}, \mathrm{OCH}_{2}\right)$, $4.16\left(\mathrm{t},{ }^{3} \mathrm{~J}=5.1 \mathrm{~Hz}, 2 \mathrm{H}, \mathrm{OCH}_{2}\right), 3.85\left(\mathrm{t},{ }^{3} \mathrm{~J}=4.9 \mathrm{~Hz}, 2 \mathrm{H}, \mathrm{OCH}_{2}\right)$, $3.30-3.18(\mathrm{~m}, 1 \mathrm{H}, \mathrm{OCH}), 2.50-0.79(\mathrm{~m}, 40 \mathrm{H}), 0.68(\mathrm{~s}, 3 \mathrm{H}, \mathrm{Me})$ ppm. ${ }^{13} \mathrm{C}$ NMR: $\delta=164.2(\mathrm{C}=\mathrm{N}), 162.0(C=\mathrm{CH}), 160.4(\mathrm{Ar}-\mathrm{C})$, 157.8 (Ar-C), 140.7 (isoxazole-CO), 134.8, 130.6, 130.6, 129.2, 126.8, 122.0, 121.8, 120.4, 116.0, 114.9, 114.8 (Ar-C, Ar-CH, and $\mathrm{C}=\mathrm{CH}), 101.2($ isoxazole- $\mathrm{CH}), 79.8(\mathrm{OCH}), 67.9\left(\mathrm{OCH}_{2}\right), 66.4$ $\left(\mathrm{OCH}_{2}\right), 61.5\left(\mathrm{CH}_{2} \mathrm{OPh}\right), 56.8,56.2,50.2,42.2,39.8,39.5,39.1$, $37.2,36.8,36.2,35.8,32.0,31.9,28.4,28.2,28.0,24.4,23.8,22.8$, 22.6, 21.1, 19.4, 18.7, 11.9 ppm. HRMS (ESI): calcd. for $\mathrm{C}_{45} \mathrm{H}_{62} \mathrm{NO}_{4}[\mathrm{M}+\mathrm{H}]^{+}$680.4673; found 680.4712 (5.7 ppm).

3-\{[3-(2-Cholest-5-en-3ß-yloxy)ethoxy]phenyl\}-5-(phenoxymethyl)isoxazole $\left(\boldsymbol{m}\right.$-18): Compound $\boldsymbol{m}-\mathbf{1 8}(31 \mathrm{mg}, 39 \%)$ was isolated. $R_{\mathrm{f}}$ $=0.44$ (hexane/EtOAc, 8:2). ${ }^{1} \mathrm{H}$ NMR: $\delta=7.39-7.10(\mathrm{~m}, 5 \mathrm{H}, \mathrm{Ar}-$ $\mathrm{H}), 7.09-6.84(\mathrm{~m}, 4 \mathrm{H}, \mathrm{Ar}-\mathrm{H}), 6.57$ (s, $1 \mathrm{H}$, isoxazole-H), 5.34 (br. $\mathrm{s}, 1 \mathrm{H}, \mathrm{CH}=\mathrm{C}), 5.17\left(\mathrm{~s}, 2 \mathrm{H}, \mathrm{OCH}_{2}\right), 4.15\left(\mathrm{t},{ }^{3} \mathrm{~J}=5.1 \mathrm{~Hz}, 2 \mathrm{H}\right.$, $\left.\mathrm{OCH}_{2}\right), 3.85\left(\mathrm{t},{ }^{3} \mathrm{~J}=5.0 \mathrm{~Hz}, 2 \mathrm{H}, \mathrm{OCH}_{2}\right), 3.32-3.19(\mathrm{~m}, 1 \mathrm{H}$, OCH), 2.50-0.79 (m, $40 \mathrm{H}), 0.68$ (s, $3 \mathrm{H}, \mathrm{Me}) \mathrm{ppm} .{ }^{13} \mathrm{C}$ NMR: $\delta$ $=168.2(\mathrm{C}=\mathrm{N}), 162.1(\mathrm{C}=\mathrm{CH}), 159.8(\mathrm{Ar}-\mathrm{C}), 159.6(\mathrm{Ar}-\mathrm{C}), 140.7$ (isoxazole-CO), 133.7, 130.5, 130.4, 129.7, 129.8, 122.1, 121.5, 118.9, 114.7, 114.6, 113.6 (Ar-C, $\mathrm{Ar}-\mathrm{CH}$, and $\mathrm{C}=\mathrm{CH}), 101.1$ (isoxazole-CH), $79.8(\mathrm{OCH}), 67.9\left(\mathrm{OCH}_{2}\right), 66.4\left(\mathrm{OCH}_{2}\right), 61.6$ $\left(\mathrm{CH}_{2} \mathrm{OPh}\right), 56.8,56.3,50.2,42.2,39.8,39.5,39.0,37.2,36.8,36.2$, $35.8,32.0,31.9,28.5,28.2,28.0,24.4,23.8,22.8,22.6,21.1,19.4$, 18.7, 11.9 ppm. HRMS (ESI): calcd. for $\mathrm{C}_{45} \mathrm{H}_{62} \mathrm{NO}_{4}[\mathrm{M}+\mathrm{H}]^{+}$ 680.4673; found 680.4706 (4.9 ppm).

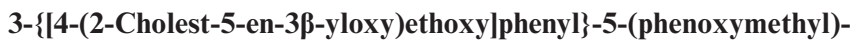
isoxazole $(p-18)$

Method A - Following the General Procedure: Compound $p$-18 was isolated in $58 \%$ yield $(47 \mathrm{mg})$.

Method B - Following a Modified Procedure: (Prop-2-yn-1-yloxy)benzene $(16 \mathrm{mg}, 0.12 \mathrm{mmol})$ was put into a round-bottomed flask, and a solution of oxime p-16 (50 mg, $0.09 \mathrm{mmol}, 0.75$ equiv.) and Ch-T (27 mg, $0.12 \mathrm{mmol}, 1$ equiv.) in $\mathrm{EtOH}(1 \mathrm{~mL}$ per $0.1 \mathrm{mmol}$ oxime) was added. The mixture was stirred for $4 \mathrm{~h}$ at room temp. The same dose of oxime/Ch-T was added again, and then the mixture was stirred for a further $17 \mathrm{~h}$ at room temp. The solvent was removed under reduced pressure, the residue was dissolved in toluene, and this solution was washed with $\mathrm{H}_{2} \mathrm{O}$. The organic layer was dried with anhydrous $\mathrm{Na}_{2} \mathrm{SO}_{4}$, and the solvent was removed under reduced pressure. The crude reaction product was purified by flash column chromatography (hexane/EtOAc 0-10\%) to give compound p-18 (65 mg, 80\%) as an off-white solid. $R_{\mathrm{f}}=0.45$ (hexane/EtOAc, 8:2). ${ }^{1} \mathrm{H}$ NMR: $\delta=7.82-7.70(\mathrm{~m}, 2 \mathrm{H}, \mathrm{Ar}-\mathrm{H}), 7.37-7.22(\mathrm{~m}, 2 \mathrm{H}$, Ar-H), 7.10-6.94 (m, 5 H, Ar-H), 6.58 (s, $1 \mathrm{H}$, isoxazole-H), 5.35 (br. s, $1 \mathrm{H}, \mathrm{CH}=\mathrm{C}), 5.19\left(\mathrm{~s}, 2 \mathrm{H}, \mathrm{OCH}_{2}\right), 4.15\left(\mathrm{t},{ }^{3} \mathrm{~J}=5.0 \mathrm{~Hz}, 2 \mathrm{H}\right.$, $\left.\mathrm{OCH}_{2}\right), 3.85\left(\mathrm{t},{ }^{3} \mathrm{~J}=4.8 \mathrm{~Hz}, 2 \mathrm{H}, \mathrm{OCH}_{2}\right), 3.32-3.18(\mathrm{~m}, 1 \mathrm{H}$, $\mathrm{OCH}), 2.50-0.78(\mathrm{~m}, 40 \mathrm{H}), 0.68(\mathrm{~s}, 3 \mathrm{H}, \mathrm{Me}) \mathrm{ppm} .{ }^{13} \mathrm{C}$ NMR: $\delta$ $=168.3(\mathrm{C}=\mathrm{N}), 162.1(C=\mathrm{CH}), 160.4($ Ar-C $), 157.8($ Ar-C), 140.8 (isoxazole-CO), 133.9, 129.8, 129.7, 128.2, 127.9, 121.9, 121.7, 121.4, 115.4, 115.0, 114.8 (Ar-C, $\mathrm{Ar}-\mathrm{CH}$, and $\mathrm{C}=\mathrm{CH}$ ), 101.1 (isoxazole- $\mathrm{CH}), 79.8(\mathrm{OCH}), 67.9\left(\mathrm{OCH}_{2}\right), 66.4\left(\mathrm{OCH}_{2}\right), 61.5$ $\left(\mathrm{CH}_{2} \mathrm{OPh}\right), 56.8,56.2,50.2,42.3,39.8,39.5,39.1,37.2,36.8,36.2$, $35.8,32.0,31.9,28.4,28.2,28.0,24.3,23.8,22.8,22.6,21.1,19.4$, $18.7,11.9$ ppm. HRMS (ESI): calcd. for $\mathrm{C}_{45} \mathrm{H}_{62} \mathrm{NO}_{4}[\mathrm{M}+\mathrm{H}]^{+}$ 680.4673; found $680.4672(-1.5 \mathrm{ppm})$.

(3ß)-3-[(4-Formyl)phenyloxy]cholest-5-ene (19): Compound 19, prepared in an analogous manner to the hydroxyethoxy analogues 15, was isolated (788 mg, 62\%) as an off-white solid. $R_{\mathrm{f}}=0.71$ (hexane/ EtOAc, 8:2). ${ }^{1} \mathrm{H}$ NMR: $\delta=9.86(\mathrm{~s}, 1 \mathrm{H}, \mathrm{CHO}), 7.81\left(\mathrm{~d},{ }^{3} J=\right.$ $8.7 \mathrm{~Hz}, 2 \mathrm{H}, \mathrm{Ar}-\mathrm{H}), 6.97\left(\mathrm{~d},{ }^{3} \mathrm{~J}=8.7 \mathrm{~Hz}, 2 \mathrm{H}, \mathrm{Ar}-\mathrm{H}\right), 5.49-5.37$ (m, $1 \mathrm{H}, \mathrm{CH}=\mathrm{C}), 4.33-4.17(\mathrm{~m}, 1 \mathrm{H}, \mathrm{OCH}), 2.56-0.77(\mathrm{~m}, 40 \mathrm{H})$, 0.69 (s, $3 \mathrm{H}, \mathrm{Me}$ ) ppm. ${ }^{13} \mathrm{C}$ NMR: $\delta=189.7(\mathrm{C}=\mathrm{O}), 163.1(\mathrm{Ar}-\mathrm{C})$, $139.8(C=\mathrm{CH}), 132.1,129.6(2 \mathrm{Ar}-\mathrm{H}), 122.9(\mathrm{Ar}-\mathrm{C}), 122.8(\mathrm{C}=\mathrm{CH})$, $115.6(2 \mathrm{Ar}-\mathrm{H}), 77.0(\mathrm{OCH}), 76.6,56.8,56.2,50.2,42.3,39.8,39.5$, $38.4,37.1,36.8,36.2,35.8,32.0,31.9,29.7,28.2,28.0,24.3,23.8$, 22.8, 22.6, 21.1, 19.4, 18.7, 11.9 ppm. HRMS (ESI): calcd. for $\mathrm{C}_{34} \mathrm{H}_{51} \mathrm{O}_{2}[\mathrm{M}+\mathrm{H}]^{+}$491.3884; found 491.3912 (5.7 ppm).

(3ß)-3-[(4-Hydroxyiminoformyl)phenyloxy]cholest-5-ene (20): Compound 20, prepared in an analogous manner to the hydroxyethoxy analogues 16, was isolated $(510 \mathrm{mg}$, quantitative) as an off-white solid. $R_{\mathrm{f}}=0.69$ (hexane/EtOAc, 8:2). ${ }^{1} \mathrm{H}$ NMR: $\delta=8.08$ (s, $1 \mathrm{H}$, $\mathrm{CH}=\mathrm{NOH}$ ), 7.85 (br. s, $1 \mathrm{H}, \mathrm{CH}=\mathrm{NOH}), 7.48\left(\mathrm{~d},{ }^{3} J=8.7 \mathrm{~Hz}, 2\right.$ $\mathrm{H}, \mathrm{Ar}-\mathrm{H}), 6.88\left(\mathrm{~d},{ }^{3} \mathrm{~J}=8.7 \mathrm{~Hz}, 2 \mathrm{H}, \mathrm{Ar}-\mathrm{H}\right), 5.48-5.33(\mathrm{~m}, 1 \mathrm{H}$, $\mathrm{CH}=\mathrm{C}), 4.25-4.06(\mathrm{~m}, 1 \mathrm{H}, \mathrm{OCH}), 2.55-0.78(\mathrm{~m}, 40 \mathrm{H}), 0.69(\mathrm{~s}, 3$ $\mathrm{H}, \mathrm{Me})$ ppm. ${ }^{13} \mathrm{C}$ NMR: $\delta=159.3(\mathrm{Ar}-\mathrm{C}), 150.0(\mathrm{C}=\mathrm{N}), 140.1$ $(C=\mathrm{CH}), 128.5(2 \mathrm{Ar}-\mathrm{H}), 124.4(\mathrm{Ar}-\mathrm{C}), 122.5(\mathrm{C}=\mathrm{CH}), 115.9(2$ Ar-H), 77.0 (OCH), 76.6, 56.8, 56.2, 50.2, 42.4, 39.8, 39.5, 38.6, $37.2,36.8,36.2,35.8,32.0,31.9,28.2,28.2,28.0,24.3,23.8,22.8$, 22.6, 21.1, 19.4, 18.7, 11.9 ppm. HRMS (ESI): calcd. for $\mathrm{C}_{34} \mathrm{H}_{52} \mathrm{NO}_{2}[\mathrm{M}+\mathrm{H}]^{+}$506.3993; found 506.4021 (5.5 ppm).

3-[(4-Cholest-5-en-3ß-yloxy)phenyloxy]-5-(phenoxymethyl)isoxazole (21): Compound 21, prepared following the modified procedure described above for $\boldsymbol{p}$-18, was isolated $(46 \mathrm{mg}, 60 \%$ ) as an off-white solid. $R_{\mathrm{f}}=0.60$ (hexane/EtOAc, 8:2). ${ }^{1} \mathrm{H}$ NMR: $\delta=7.80\left(\mathrm{~d},{ }^{3} J=\right.$ $8.4 \mathrm{~Hz}, 2 \mathrm{H}, \mathrm{Ar}-\mathrm{H}), 7.71\left(\mathrm{~d},{ }^{3} J=8.4 \mathrm{~Hz}, 2 \mathrm{H}, \mathrm{Ar}-\mathrm{H}\right), 7.37-7.23$ (m, $2 \mathrm{H}, \mathrm{Ar}-\mathrm{H}), 7.05-6.91(\mathrm{~m}, 3 \mathrm{H}, \mathrm{Ar}-\mathrm{H}), 6.58(\mathrm{~s}, 1 \mathrm{H}$, isoxazole$\mathrm{H}), 5.33-5.48(\mathrm{~m}, 1 \mathrm{H}, \mathrm{CH}=\mathrm{C}), 5.19\left(\mathrm{~s}, 2 \mathrm{H}, \mathrm{OCH}_{2}\right), 4.25-4.08(\mathrm{~m}$, $1 \mathrm{H}, \mathrm{OCH}), 2.50-0.78(\mathrm{~m}, 40 \mathrm{H}), 0.69(\mathrm{~s}, 3 \mathrm{H}, \mathrm{Me}) \mathrm{ppm} .{ }^{13} \mathrm{C} \mathrm{NMR}$ : $\delta=168.2(\mathrm{C}=\mathrm{N}), 162.1(C=\mathrm{CH}), 159.7,159.6(2 \mathrm{Ar}-\mathrm{C}), 140.6$ (isoxazole-CH), 133.6, 130.5, 130.4, 129.8, 129.8, 122.1, 121.5, 118.9, 114.7, 114.6, 113.6 (Ar-C, $9 \mathrm{Ar}-\mathrm{CH}$, and $\mathrm{C}=\mathrm{CH}), 101.1$ (isoxazole$\mathrm{CH}), 79.8(\mathrm{OCH}), 61.5\left(\mathrm{CH}_{2} \mathrm{OPh}\right), 56.8,56.2,50.2,42.2,39.8,39.5$, $39.0,37.3,36.8,36.2,35.8,32.0,31.9,28.4,28.2,28.0,24.4,23.8$, 22.8, 22.6, 21.1, 19.4, 18.7, 11.9 ppm. HRMS (ESI): calcd. for $\mathrm{C}_{43} \mathrm{H}_{58} \mathrm{NO}_{3}[\mathrm{M}+\mathrm{H}]^{+}$636.4411; found 636.4449 (6.0 ppm).

General Procedure for the Nitrile Oxide-Alkyne Cycloaddition with Sugar and Nucleoside Substrates: Alkylated sugar 22[21] ( 0.75 equiv.), thymidine mono-alkyne 24 ( 0.75 equiv.), or thymidine bis-alkyne $\mathbf{2 6}^{[18]}$ (1.5 equiv.) was put into a round-bottomed flask, and a solution of oxime $\mathbf{p - 1 6}$ and Ch-T (1.5 equiv. for $\mathbf{2 2}$ and $\mathbf{2 4 ,}$ 
and 3.0 equiv. for 26) in $\mathrm{EtOH}$ ( $2 \mathrm{~mL}$ per $0.1 \mathrm{mmol}$ oxime) was added. The mixture was stirred for $4 \mathrm{~h}$ at room temp. The same dose of oxime/Ch-T was added again, and then the mixture was stirred for a further $17 \mathrm{~h}$ at room temp. The solvent was removed under reduced pressure. The residue was dissolved in $\mathrm{CH}_{2} \mathrm{Cl}_{2}$, and this solution was washed with $\mathrm{H}_{2} \mathrm{O}$. The organic layer was dried with anhydrous sodium sulfate, and the solvent was removed under reduced pressure. The crude product was purified as described below.

3-\{4-[2-(Cholest-5-en-3ß-yloxy)ethoxy]phenyl\}-5-(6-O-\{[1,2:3,4-bis$O$-(1-methylethylidene)- $\alpha$-D-galactopyranose]methoxymethyl $\}$ isoxazole) (23): The crude reaction product was purified by flash column chromatography (hexane/EtOAc 10-35\%) to give compound $23(118 \mathrm{mg}, 85 \%)$ as a white sticky solid. $R_{\mathrm{f}}=0.35$ (hexane/ EtOAc, 7:3). ${ }^{1} \mathrm{H}$ NMR: $\delta=7.92-7.80(\mathrm{~m}, 2 \mathrm{H}$, Ar-H), 7.07-6.99 $(\mathrm{m}, 2 \mathrm{H}, \mathrm{Ar}-\mathrm{H}), 6.54\left(\mathrm{~s}, 1 \mathrm{H}\right.$, isoxazole-H), $5.54\left(\mathrm{~d},{ }^{3} \mathrm{~J}=5.7 \mathrm{~Hz}, 1\right.$ $\left.\mathrm{H}, 1^{\prime}-\mathrm{H}\right), 5.34$ (br. s, $\left.1 \mathrm{H}, \mathrm{CH}=\mathrm{C}\right), 5.10\left(\mathrm{~s}, 2 \mathrm{H}, \mathrm{OCH}_{2}\right), 4.61\left(\mathrm{~d},{ }^{3} \mathrm{~J}\right.$ $\left.=5.7 \mathrm{~Hz}, 1 \mathrm{H}, 2^{\prime}-\mathrm{H}\right), 4.31-4.14\left(\mathrm{~m}, 4 \mathrm{H}, 3^{\prime}-\mathrm{H}, 4^{\prime}-\mathrm{H}, \mathrm{OCH}_{2}\right), 4.07-$ $3.96\left(\mathrm{~m}, 3 \mathrm{H}, 5^{\prime}-\mathrm{H}, \mathrm{OCH}_{2}\right), 3.82-3.73\left(\mathrm{~m}, 1 \mathrm{H}, 6^{\prime}-\mathrm{H}\right), 3.73-3.63$ $\left(\mathrm{m}, 1 \mathrm{H}, 6^{\prime}-\mathrm{H}\right), 3.32-3.15(\mathrm{~m}, 1 \mathrm{H}, \mathrm{OCH}), 2.36-0.81(\mathrm{~m}, 52 \mathrm{H})$, $0.68(\mathrm{~s}, 3 \mathrm{H}, \mathrm{Me}) \mathrm{ppm} .{ }^{13} \mathrm{C} \mathrm{NMR}: \delta=167.3(\mathrm{C}=\mathrm{N}), 162.0(\mathrm{C}=\mathrm{CH})$, 159.8 (isoxazole-CO), $140.8(\mathrm{Ar}-\mathrm{C}), 132.0(\mathrm{Ar}-\mathrm{CH}), 130.2(\mathrm{C}=\mathrm{CH})$, 121.6 (Ar-C), $114.8(\mathrm{Ar}-\mathrm{CH}), 109.3,108.6\left[\mathrm{C}\left(\mathrm{CH}_{3}\right)_{2}\right], 100.9$ (isox-

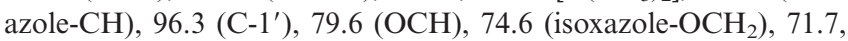
$71.2,70.7,70.5\left(\mathrm{C}-2^{\prime}, \mathrm{C}-3^{\prime}, \mathrm{C}-4^{\prime}, \mathrm{C}-5^{\prime}\right), 69.6\left(\mathrm{OCH}_{2}\right), 68.7\left(\mathrm{OCH}_{2}\right)$, $66.7\left(\mathrm{C}-6^{\prime}\right), 61.0\left(\mathrm{OCH}_{2}\right), 58.5,56.7,56.1,50.1,42.3,39.8,39.5$, $37.2,36.5,36.2,35.8,31.9,31.6,28.2,28.0,26.0,24.9,24.5,24.3$, $24.0,23.8,22.8,22.6,21.1,19.4,18.7,11.9$ ppm. IR: $\tilde{v}=1562$, 1409, 1330, 1245, 1160, 1019, $832 \mathrm{~cm}^{-1}$. HRMS (ESI): calcd. for $\mathrm{C}_{51} \mathrm{H}_{76} \mathrm{NO}_{9}[\mathrm{M}+\mathrm{H}]^{+}$846.5515; found 846.5558 (5.1 ppm).

$3^{\prime}$-O-\{(3)-4-Ethoxy-[2-(Cholest-5-en-3ß-yloxy)phenyl]isoxazol-5yl\}methyl-5'-O-[bis(4-methoxyphenyl)phenylmethyl]thymidine (25): The crude reaction product was purified by flash column chromatography (hexane/EtOAc 20-40\%) to give compound $\mathbf{2 5}$ $(53 \mathrm{mg}, 81 \%)$ as an off-white gum. $R_{\mathrm{f}}=0.30$ (hexane/EtOAc, 7:3). ${ }^{1} \mathrm{H}$ NMR: $\delta=8.32$ (br. s, $\left.1 \mathrm{H}, \mathrm{NH}\right), 7.91-7.83$ (m, $\left.2 \mathrm{H}, \mathrm{Ar}-\mathrm{H}\right)$, 7.63-7.58 (m, $1 \mathrm{H}, \mathrm{Ar}-\mathrm{H}), 7.47-7.23$ (m, $2 \mathrm{H}, \mathrm{Ar}-\mathrm{H}), 7.10-7.03$ (m, $2 \mathrm{H}, \mathrm{Ar}-\mathrm{H}), 6.92-6.83(\mathrm{~m}, 4 \mathrm{H}, \mathrm{Ar}-\mathrm{H}), 6.56(\mathrm{~s}, 1 \mathrm{H}$, isoxazole- $\mathrm{H})$, $6.44\left(\mathrm{t},{ }^{3} J=6.9 \mathrm{~Hz}, 1 \mathrm{H}, 1^{\prime}-\mathrm{H}\right), 5.39$ (br. s, $\left.1 \mathrm{H}, \mathrm{CH}=\mathrm{C}\right), 4.66-4.58$ $\left(\mathrm{m}, 1 \mathrm{H}, 3^{\prime}-\mathrm{H}\right), 4.52\left(\mathrm{~s}, 2 \mathrm{H}, \mathrm{OCH}_{2}\right), 4.23\left(\mathrm{t},{ }^{3} \mathrm{~J}=13.9 \mathrm{~Hz}, 2 \mathrm{H}\right.$, $\left.\mathrm{OCH}_{2}\right), 4.12-4.06\left(\mathrm{~m}, 1 \mathrm{H}, 4^{\prime}-\mathrm{H}\right), 3.90\left(\mathrm{t},{ }^{3} \mathrm{~J}=13.9 \mathrm{~Hz}, 2 \mathrm{H}\right.$, $\left.\mathrm{OCH}_{2}\right), 3.83(\mathrm{~m}, 6 \mathrm{H}, 2 \mathrm{OMe}), 3.57-3.24\left(\mathrm{~m}, 3 \mathrm{H}, \mathrm{OCH}, 25^{\prime}-\mathrm{H}\right)$, $2.50-0.82(\mathrm{~m}, 45 \mathrm{H}), 0.72(\mathrm{~s}, 3 \mathrm{H}, \mathrm{Me}) \mathrm{ppm} .{ }^{13} \mathrm{C} \mathrm{NMR}: \delta=170.0$ $(\mathrm{C}=\mathrm{N}), 163.4(\mathrm{C}-2), 161.9(\mathrm{C}=\mathrm{CH}), 159.7$ (isoxazole-CO), 150.1 (C-4), 140.2 (Ar-C), 135.3 (C-6), 132.0, 130.2, 130.1, 129.1, 128.6, $128.4,128.1,127.4,126.9,121.1,113.8(\mathrm{Ar}-\mathrm{C}, \mathrm{Ar}-\mathrm{CH}, \mathrm{C}=\mathrm{CH})$, 111.2 (C-5), 102.3 (isoxazole- $\mathrm{CH}), 86.5\left(\mathrm{CPh}_{3}\right), 83.9\left(\mathrm{C}-1^{\prime}\right), 83.8$ $\left(\mathrm{C}-4^{\prime}\right), 80.1\left(\mathrm{C}-3^{\prime}\right), 79.6(\mathrm{OCH}), 74.5\left(\right.$ isoxazole- $\left.\mathrm{OCH}_{2}\right), 69.6$ $\left(\mathrm{OCH}_{2}\right), 68.9\left(\mathrm{OCH}_{2}\right), 63.8\left(\mathrm{C}-5^{\prime}\right), 62.3\left(\mathrm{OCH}_{2}\right), 58.8,56.5,56.1$, 50.1, 42.4, 39.8, 39.6, 37.8, 37.3, 36.5, 35.8, 31.8, 31.6, 28.2, 28.1, $26.0,24.9,24.4,24.0,23.7,22.8,21.1,19.9,18.4,11.9$ ppm. IR: ̃ $=3310,1709,1570,1411,1350,1229,1021,848 \mathrm{~cm}^{-1}$. HRMS (ESI): calcd. for $\mathrm{C}_{70} \mathrm{H}_{88} \mathrm{~N}_{3} \mathrm{O}_{10}[\mathrm{M}+\mathrm{H}]^{+} 1130.6464$; found $1130.6525(5.4 \mathrm{ppm})$.

$3^{\prime}-O, N^{3}$-Bis-\{(3)-4-Ethoxy-[2-(Cholest-5-en-3ß-yloxy)phenyl]isoxazol-5-yl\}methyl]-5' $-O$-[bis(4-methoxyphenyl)phenylmethyl]thymidine (27): The crude reaction product was purified by flash column chromatography (hexane/EtOAc $10-30 \%$ ) to give compound 27 (64 mg, 68\%) as an off-white gum. $R_{\mathrm{f}}=0.45$ (hexane/EtOAc, 7:3). ${ }^{1} \mathrm{H}$ NMR: $\delta=7.87-7.79(\mathrm{~m}, 4 \mathrm{H}, \mathrm{Ar}-\mathrm{H}), 7.59-7.56(\mathrm{~m}, 1 \mathrm{H}$, ArH), 7.42-7.20 (m, 8 H, Ar-H), 7.05-6.99 (m, 4 H, Ar-H), 6.88-6.80 $(\mathrm{m}, 4 \mathrm{H}, \mathrm{Ar}-\mathrm{H}), 6.58(\mathrm{~s}, 1 \mathrm{H}$, isoxazole- $\mathrm{H}), 6.53(\mathrm{~s}, 1 \mathrm{H}$, isoxazole-
$\mathrm{H}), 6.42\left(\mathrm{t},{ }^{3} \mathrm{~J}=6.9 \mathrm{~Hz}, 1 \mathrm{H}, 1^{\prime}-\mathrm{H}\right), 5.47-5.28(\mathrm{~m}, 3 \mathrm{H}, \mathrm{CH}=\mathrm{C}$, $\left.\mathrm{NCH}_{2}\right), 4.58-4.42\left(\mathrm{~m}, 3 \mathrm{H}, 3^{\prime}-\mathrm{H}, \mathrm{OCH}_{2}\right), 4.23-4.15(\mathrm{~m}, 4 \mathrm{H}, 2$ $\left.\mathrm{OCH}_{2}\right), 4.07-4.02\left(\mathrm{~m}, 1 \mathrm{H}, 4^{\prime}-\mathrm{H}\right), 3.90-3.82\left(\mathrm{~m}, 4 \mathrm{H}, 2 \mathrm{OCH}_{2}\right) 3.79$ (m, $6 \mathrm{H}, 2 \mathrm{OMe}), 3.52-3.21\left(\mathrm{~m}, 4 \mathrm{H}, 2 \mathrm{OCH}, 25^{\prime}-\mathrm{H}\right), 2.46-0.78$ $(\mathrm{m}, 85 \mathrm{H}), 0.68(\mathrm{~s}, 6 \mathrm{H}, 2 \mathrm{Me}) \mathrm{ppm} .{ }^{13} \mathrm{C} \mathrm{NMR}: \delta=169.8(\mathrm{C}=\mathrm{N})$, $168.1(\mathrm{C}=\mathrm{N}), 163.0(\mathrm{C}-2), 161.9(2 \mathrm{C}=\mathrm{CH}), 159.7(2$ isoxazole- $\mathrm{CO})$, 150.1 (C-4), 142.8 (Ar-C), 135.0 (C-6), 132.0, 130.2, 130.1, 129.2, 128.7, 128.6, 128.1, 127.5, 126.9, 121.6, 114.8 (Ar-C, Ar-CH, 2 $\mathrm{C}=\mathrm{CH}), 111.2(\mathrm{C}-5), 102.3(2$ isoxazole- $\mathrm{CH}), 87.6\left(\mathrm{CPh}_{3}\right), 85.1(\mathrm{C}-$ $\left.1^{\prime}\right), 83.8\left(\mathrm{C}-4^{\prime}\right), 80.1\left(\mathrm{C}-3^{\prime}\right), 79.6(2 \mathrm{OCH}), 74.5$ (2 isoxazole$\left.\mathrm{OCH}_{2}\right), 69.6\left(2 \mathrm{OCH}_{2}\right), 68.9\left(2 \mathrm{OCH}_{2}\right), 63.8\left(\mathrm{C}-5^{\prime}\right), 62.3\left(\mathrm{OCH}_{2}\right)$, $58.5,56.5,56.1,50.1,42.5,39.8,39.6,37.9,37.3,36.5,36.2,35.9$, $31.8,31.6,28.2,26.1,24.8,24.4,24.0,23.7,22.8,22.7,21.1,19.4$, $18.7,11.9$ ppm. IR: $\tilde{v}=1699,1580,1411,1335,1223,1021$, $838 \mathrm{~cm}^{-1}$. HRMS (ESI): calcd. for $\mathrm{C}_{109} \mathrm{H}_{143} \mathrm{~N}_{4} \mathrm{O}_{13}[\mathrm{M}+\mathrm{H}]^{+}$ 1716.0646; found 1716.0741 (5.5 ppm).

Supporting Information (see footnote on the first page of this article): Copies of ${ }^{1} \mathrm{H}$ and ${ }^{13} \mathrm{C}$ NMR spectra of new compounds.

\section{Acknowledgments}

The work was supported by the Science Foundation of Ireland (programme code 05/PICA/B838/NSs) and the Irish Research Council of Science and Engineering (Embark Postgraduate Research Scholarship to JOS, programme code RS/2012/128)

[1] V. V. Rostovtsev, L. G. Green, V. V. Fokin, K. B. Sharpless, Angew. Chem. Int. Ed. 2002, 41, 2596-2599; Angew. Chem. 2002, 114, 2708-2711; C. W. Tornoe, C. Christensen, M. Meldal, J. Org. Chem. 2002, 67, 3057-3064.

[2] a) J. A. Lenhart, X. Ling, R. Gandhi, T. L. Guo, P. M. Gerk, D. H. Brunzell, S. Zhang, J. Med. Chem. 2010, 53, 6198-6209; b) F. Alonso, Y. Moglie, G. Radivoy, M. Yus, Heterocycles 2012, 84, 1033-1044; c) D. D. Díaz, J. J. Cid, P. Vázquez, T. Torres, Chem. Eur. J. 2008, 14, 9261-9273; d) G. Godeau, P. Barthelemy, Langmuir 2009, 25, 8447-8450; e) S. Karsten, M. A. Ameen, S. I. Kallaene, A. Nan, R. Turcu, J. Liebscher, Synthesis 2010, 3021-3028; f) K. C. Majumdar, S. Mondal, R. K. Sinha, New J. Chem. 2010, 34, 1255-1260; g) X.-M. Liu, Y. Zhang, F. Chen, I. Khutsishvili, E. V. Fehringer, L. A. Marky, K. W. Bayles, D. Wang, Pharm. Res. 2012, 29, 3169 3179; h) Z. Li, R. Bittman, J. Org. Chem. 2007, 72, 8376-8382; i) C. Thiele, C. Papan, D. Hoelper, K. Kusserow, A. Gaebler, M. Schoene, K. Piotrowitz, D. Lohmann, J. Spandl, A. Stevanovic, A. Shevchenko, L. Kuerschner, ACS Chem. Biol. 2012, 7, 2004-2011; j) A. M. Deobald, L. R. S. Camargo, D. Alves, J. Zukerman-Schpector, A. G. Correa, M. W. Paixao, Synthesis 2011, 4003-4010; k) G. Godeau, C. Staedel, P. Barthelemy, J. Med. Chem. 2008, 51, 4374 4376; 1) T. Yamada, C. G. Peng, S. Matsuda, H. Addepalli, K. N. Jayaprakash, M. R. Alam, K. Mills, M. A. Maier, K. Charisse, M. Sekine, M. Manoharan, K. G. Rajeev, J. Org. Chem. 2011, 76, 1198-1211; m) J. Willibald, J. Harder, K. Sparrer, K.-K. Conzelmann, T. Carell, J. Am. Chem. Soc. 2012, 134, 12330-12333.

[3] I. Singh, C. Freeman, F. Heaney, Eur. J. Org. Chem. 2011, 6739-6746.

[4] a) V. A. Khripach, V. N. Zhabinskii, D. N. Tsavlovskii, O. A. Drachenova, G. V. Ivanova, O. V. Konstantinova, M. I. Zavadskaya, A. S. Lyakhov, A. A. Govorova, M. B. Groen, A. de Groot, Steroids 2001, 66, 569-579; b) F. Jourdan, C. Bubert, M. P. Leese, A. Smith, E. Ferrandis, S. Regis-Lydi, S. P. Newman, A. Purohit, M. J. Reed, B. V. L. Potter, Org. Biomol. Chem. 2008, 6, 4108-4119; c) A. A. Akhrem, V. A. Khripach, F. A. Lakhvich, M. I. Zavadskaya, O. A. Drachenova, I. A. Zorina, Dokl. Akad. Nauk SSSR 1987, 297, 364-367 [Chem. Abstr. 108, 221953]; d) A. A. Akhrem, V. A. Khripach, F. A. 
Lakhvich, M. I. Zavadskaya, O. A. Drachenova, I. A. Zorina, Zh. Org. Khim. 1989, 25, 2120-2128 [Chem. Abstr. 113, 24322]; e) R. P. Litvinovskaya, S. V. Drach, V. A. Khripach, Russ. J. Org. Chem. 2001, 37, 787-792.

[5] a) D. Kovacs, Z. Kadar, G. Motyan, G. Schneider, J. Wolfling, I. Zupko, E. Frank, Steroids 2012, 77, 1075-1085; b) F. Himo, T. Lovell, R. Hilgraf, V. V. Rostovtsev, L. Noodleman, K. B. Sharpless, V. V. Fokin, J. Am. Chem. Soc. 2005, 127, 210-216; c) T. V. Hansen, P. Wu, V. V. Fokin, J. Org. Chem. 2005, 70, 7761-7764; d) S. Grecian, V. V. Fokin, Angew. Chem. Int. Ed. 2008, 47, 8285-8287; Angew. Chem. 2008, 120, 8409-8411.

[6] a) V. V. Vaidya, K. S. Wankhede, S. J. Nara, M. M. Salunkhe, G. K. Trivedi, Synth. Commun. 2009, 39, 3856-3866; b) K. S. Wankhede, V. V. Vaidya, P. S. Sarang, M. M. Salunkhe, G. K. Trivedi, Tetrahedron Lett. 2008, 49, 2069-2073; c) A. V. Baranovsky, D. A. Bolibrukh, J. R. Bull, Eur. J. Org. Chem. 2007, 445-454.

[7] a) V. A. Khripach, V. N. Zhabinskii, A. I. Kotyatkina, Zh. Org. Khim. 1993, 29, 1573-1577 [Chem. Abstr. 121, 231146]; b) B. Jiang, Y. Liu, W.-S. Zhou, J. Org. Chem. 2000, 65, 6231-6236; c) R. P. Litvinovskaya, S. V. Drach, V. A. Khripach, Bioorg. Khim. 1998, 24, 236-240 [Chem. Abstr. 131, 73840].

[8] F. Heaney, Eur. J. Org. Chem. 2012, 3043-3058.

[9] B. Lu, L.-J. Li, T.-S. Li, J.-T. Li, J. Chem. Res. Synop. 1998, 604-605.

[10] M. Rega, C. Jimenez, J. Rodriguez, Steroids 2007, 72, 729-735.

[11] G. Gimpl, K. Gehrig-Burger, Steroids 2011, 76, 216-231.

[12] a) P.-C. Yang, Y.-L. Lu, C.-Y. Li, J. Mol. Struct. 2012, 1015, 129-137; b) K. Murata, M. Aoki, T. Nishi, A. Ikeda, S. Shinkai, J. Chem. Soc., Chem. Commun. 1991, 1715-1718; c) X.-M.
Liu, B. Yang, Y.-L. Wang, J.-Y. Wang, Biochim. Biophys. Acta Biomembr. 2005, 1720, 28-34.

[13] a) I. Singh, F. Heaney, Chem. Commun. 2011, 47, 2706-2708; b) C. Freeman, J. S. Vyle, F. Heaney, RSC Adv. 2013, 3, 16521655.

[14] a) P.-Y. Jin, P. Jin, Y.-A. Ruan, Y. Ju, Y.-F. Zhao, Synlett 2007, 3003-3006; b) A. Barnard, P. Posocco, S. Pricl, M. Calderon, R. Haag, M. E. Hwang, V. W. T. Shum, D. W. Pack, D. K. Smith, J. Am. Chem. Soc. 2011, 133, 20288-20300.

[15] M.-O. Simon, S. A. Girard, C.-J. Li, Angew. Chem. Int. Ed. 2012, 51, 7537-7540; Angew. Chem. 2012, 124, 7655-7658.

[16] a) T. Masuda, H. Akita, T. Nishio, K. Niikura, K. Kogure, K. Ijiro, H. Harashima, Biomaterials 2008, 29, 709-723; b) K.-m. Choi, I. C. Kwon, H. J. Ahn, Biomaterials 2013, 34, 4183-4190; c) O. Kaczmarek, H. A. Scheidt, A. Bunge, D. Föse, S. Karsten, A. Arbuzova, D. Huster, J. Liebscher, Eur. J. Org. Chem. 2010, 1579-1586; d) A. S. Achalkumar, R. J. Bushby, S. D. Evans, Soft Matter 2010, 6, 6036-6051.

[17] K. S. Wankhede, V. V. Vaidya, P. S. Sarang, M. M. Salunkhe, G. K. Trivedi, Tetrahedron Lett. 2008, 49, 2069-2073.

[18] V. Algay, I. Singh, F. Heaney, Org. Biomol. Chem. 2010, 8, 391397.

[19] T. Ishi-i, R. Iguchi, E. Snip, M. Ikeda, S. Shinkai, Langmuir 2001, 17, 5825-5833.

[20] M. G. Simpson, M. Pittelkow, S. P. Watson, J. K. M. Sanders, Org. Biomol. Chem. 2010, 8, 1181-1187.

[21] H. B. Mereyala, S. R. Gurrala, S. K. Mohan, Tetrahedron 1999, $55,11331-11342$.

Received: December 6, 2013 Published Online: February 24, 2014 